Full length article

\title{
In situ investigation of stress-induced martensitic transformation in granular shape memory ceramic packings ${ }^{\text {is }}$
}

\author{
Hunter A. Rauch a, Yan Chen ${ }^{\text {b }}$, Ke An ${ }^{\text {b }, ~ H a n g ~ Z . ~ Y u ~ a, ~ * ~}$ \\ a Department of Materials Science and Engineering, Virginia Tech, 445 Old Turner Street, Blacksburg, VA, 24061, USA \\ ${ }^{\mathrm{b}}$ Neutron Scattering Division, Oak Ridge National Laboratory, 1 Bethel Valley Rd, Oak Ridge, TN, 37830, USA
}

\section{A R T I C L E I N F O}

\section{Article history:}

Received 30 September 2018

Received in revised form

15 February 2019

Accepted 16 February 2019

Available online 22 February 2019

\section{Keywords:}

Shape memory

Zirconia

Granular materials

Martensitic transformation

Neutron diffraction

\begin{abstract}
A B S T R A C T
Stress-induced martensitic transformation can occur in granular shape memory materials when individual particles experience high stresses and transform from a high-symmetry austenite phase to a lowsymmetry martensite phase. This involves a highly heterogeneous distribution of the driving force and very low mechanical constraint for martensite nucleation, so the transformation behavior can be dramatically different from the well-documented case of monolithic solids. In this work, we investigate the stress-induced martensitic transformation in granular shape memory ceramic packings, which consist of single-crystal micro-particles of $\mathrm{ZrO}_{2}-12$ at $\% \mathrm{CeO}_{2}$ and $\mathrm{ZrO}_{2}-15$ at $\% \mathrm{CeO}_{2}$. Through in situ neutron diffraction, we study how the phase fraction, lattice strain, and integral peak broadness evolve during external loading, unloading, and subsequent heating. Several peculiar features are discovered, including (i) a continuous mode of transformation with a wide range of transformation loads, (ii) co-evolution of the packing structure, contact deformation, and martensitic transformation, and (iii) a strong correlation between the peak broadening and the transformed phase fraction. In addition, we show the first direct evidence of reversible stress-induced martensitic transformation in granular materials. We finally discuss the mechanism for martensite nucleation and growth in granular packings and show how that leads to the observed transformation characteristics.
\end{abstract}

๑ 2019 Acta Materialia Inc. Published by Elsevier Ltd. All rights reserved.

\section{Introduction}

A diffusionless phase transformation between two crystal structures, martensitic transformation is characterized by a sheardominant shape change with the kinetics and morphology determined by shear-strain energy [1]. The forward martensitic transformation from the austenite phase to the martensite phase can be triggered by various external stimuli, including temperature, stress, and electromagnetic fields [2-6]. Stress-induced martensitic

\footnotetext{
is This manuscript has been authored by UT-Battelle, LLC under Contract No. DEAC05-000R22725 with the U.S. Department of Energy. The United States Government retains and the publisher, by accepting the article for publication, acknowledges that the United States Government retains a non-exclusive, paid-up, irrevocable, worldwide license to publish or reproduce the published form of this manuscript, or allow others to do so, for United States Government purposes. The Department of Energy will provide public access to these results of federally sponsored research in accordance with the DOE Public Access Plan (http://energy. gov/downloads/doe-public-access-plan).

* Corresponding author.

E-mail address: hangyu@vt.edu (H.Z. Yu).
}

transformation (SIMT) is responsible for several remarkable effects, such as transformation-induced plasticity, transformation toughening [7-9], and, of particular interest, shape memory and superelasticity [10-14]. The macroscopic characteristics of SIMT are defined by two key factors: the thermodynamic driving force that is dictated by the temperature and stress state, and the martensite nucleation barrier that is critically dependent on the mechanical constraint [15]. For monolithic solids, the SIMT behavior is well documented with regards to the transformation reversibility, forward and reverse critical stresses, and plateaus in the stress-strain curve due to the transformation shear strain [2,16,17].

Compared to monolithic solids, the features of thermodynamic driving force and nucleation barrier are fundamentally different in a peculiar material form: granular packings. Granular materials are complex organizations of individual particles with a distribution of the shape and size that determines their properties, and have demonstrated intriguing mechanical behavior [18-22]. In a granular packing, the driving force for SIMT is highly heterogeneous because of the non-uniform stress distributions that arise across multiple length scales. On the mesoscale, force chain formation and 
contact network evolution lead to preferred stress transmission paths [21,22]; on the microscale, contact deformation leads to stress concentrations close to the inter-particle contact sites [23], with comparatively low stresses in the particle centers. The martensite nucleation barrier is much lower in a granular packing than a monolithic solid because the mechanical constraint is significantly reduced thanks to each particle's ability to access the free surface. Furthermore, the latent heat released by SIMT, despite being an intrinsic material property, can have different effects on granular packings and monolithic solids. Inside a monolithic solid, the released heat from local transformation events may cause adjacent regions to temporarily raise temperature and thus decrease the thermodynamic driving force for SIMT, especially if the thermal conductivity of the solid is low. For a granular packing, in contrast, the released heat by local transformation can be easily dissipated through air convection because of the high surface area and small particle size. This generally precludes dynamic temperature gradients in a transforming granular packing and further emphasizes the localized nature of SIMT in this case. These features separate granular packings from monolithic solids in such dramatic fashion that conspicuously new martensitic transformation behavior may arise, which could be of great scientific interest.

Understanding SIMT in granular packings is also of practical significance because the granular form provides a pathway to using brittle smart materials in bulk applications. For example, the brittle $\mathrm{ZrO}_{2}-\mathrm{CeO}_{2}$ shows excellent shape memory and superelastic performances at small scales [12,24-26], but is hard to deploy in the traditional ceramic form (i.e., monolithic polycrystal) owing to its susceptibility to fracture during SIMT [27]. In the granular packing form, fracture reduces the particle size and is much less deleterious because the fractured particles may still be able to undergo SIMT during further mechanical loading as long as the new particle size is above a critical value. Recently, Yu and coworkers have confirmed that significant SIMT can occur in $\mathrm{ZrO}_{2}-\mathrm{CeO}_{2}$ shape memory ceramic packings by examining the metastable austenite phase via ex situ characterization [28]. This prior work specifically supports the feasibility of using the granular packing form to scale shape memory ceramics for bulk-level energy dissipation applications.

However, the exact transformation characteristics and mechanisms in granular packings are still unclear. First, ex situ characterization in previous work does not offer transformation information during unloading [28], so the questions remain regarding how much of the transformed phase reverses during unloading and whether reversible SIMT is possible in granular packings. Second, because of the complicated dynamic microstructure evolution with load [29], many transformation characteristics cannot be derived by simply examining the loaddisplacement curves. These issues can only be addressed by in situ characterization [30-34], which requires mechanically robust encapsulation of the packings and the use of penetrating beams.

Here, we employ in situ neutron diffraction to study the SIMT characteristics of granular packings, which consist of single crystal, micro-scale $\mathrm{ZrO}_{2}-\mathrm{CeO}_{2}$ particles. By investigating the evolution of phase fraction, lattice strain, and peak broadness during external loading, unloading, and subsequent heating, we find several peculiar features of SIMT in granular packings. These include (i) a continuous mode of transformation over a wide range of loads, (ii) co-evolution of the packing structure, contact deformation, and martensitic transformation, and (iii) a strong correlation between peak broadening and the transformed phase fraction in SIMT. We also show the first direct evidence of reversible SIMT in granular materials. Finally, we discuss the mechanism of martensite nucleation and growth in granular packings, with emphasis on influences of local stress concentration and free surface. Based on the discussion, we are able to explain the observed transformation characteristics.

Note that, by using single crystal particle-based shape memory ceramic packings, we preclude potential interfering effects in neutron diffraction signals, such as plasticity, precipitates, or inclusions that would exist in shape memory alloys [35], and the intra-particle mechanical constraint that would exist in polycrystalline particles. Therefore, the observed transformation characteristics mostly reflect the effects of the special structural arrangement in granular shape memory materials: heterogenous driving force and low barrier for martensite nucleation.

\section{Experimental procedures}

\subsection{Materials synthesis and characterization}

Single crystal shape memory ceramic particles were synthesized with two compositions, $\mathrm{ZrO}_{2}-12$ at $\% \mathrm{CeO}_{2}$ and $\mathrm{ZrO}_{2}-15$ at $\% \mathrm{CeO}_{2}$, both of which can transform from tetragonal (austenite) to monoclinic (martensite) structures under stress. $\mathrm{ZrO}_{2}-12$ at $\% \mathrm{CeO}_{2}$ corresponds to the metastable austenite phase with irreversible SIMT (intermediate regime), whereas $\mathrm{ZrO}_{2}-15$ at $\% \mathrm{CeO}_{2}$ corresponds to the stable austenite phase with reversible SIMT (superelastic regime) $[24,28]$. The specific transformation behavior depends on the relationship between room temperature $T_{0}$ and the transformation temperatures, $M_{F}$ (martensite finish), $M_{S}$ (martensite start), $A_{S}$ (austenite start), and $A_{F}$ (austenite finish).

The powders were fabricated by co-precipitation followed by calcination, high energy ball milling, and annealing, with the synthesis procedures detailed in Ref. [28]. The synthesized particle size ranged from 1 to $5 \mu \mathrm{m}$. Its distribution was confirmed to be monomodal by dynamic light scattering (Horiba LA 950) and scanning electron microscopy (SEM, Helios Nanolab 600 DualBeam). In the latter, dispersed particles were sputtered with $7 \mathrm{~nm}$ of $\mathrm{Pt} / \mathrm{Pd}$ and imaged on carbon tape, and showed no grain boundaries in individual particles. Post-annealing X-ray diffraction (XRD, Panalytical $\mathrm{X}^{\prime}$ Pert ${ }^{3}$ ) measurements showed that both the $\mathrm{ZrO}_{2}-12$ at\% $\mathrm{CeO}_{2}$ and $\mathrm{ZrO}_{2}-15$ at\% $\mathrm{CeO}_{2}$ packings were $96-97 \mathrm{vol} \%$ austenite (tetragonal) before testing, with the remainder being martensite (monoclinic). The small percentage of martensite in these packings may be attributed to the pinning of martensite plates by dislocations that are introduced during high energy ball milling. Dislocations are known to facilitate the formation of these plates, and could increase the reverse martensitic transformation temperature for a pinned plate considerably [2,12]. Fig. 1(a) shows examples of the synthesized single crystal austenitic particles, and Fig. 1(b) shows XRD two-theta scan patterns of the synthesized packings.

\subsection{In situ neutron diffraction experiments}

In situ neutron diffraction experiments were performed using a time-of-flight diffractometer, Vulcan, at Oak Ridge National Laboratory [36]. To dynamically observe the SIMT in granular packings [34], an encapsulated die consisting of two high-strength maraging steel rams and an aluminum or steel sleeve was employed (diagram shown in Fig. 2(a)). Since the critical stress for SIMT in monolithic $\mathrm{ZrO}_{2}-\mathrm{CeO}_{2}$ is on the order of $1 \mathrm{GPa}$, maraging steel was used to sustain high applied stresses. The cavity of the die was filled with the synthesized $\mathrm{ZrO}_{2}-\mathrm{CeO}_{2}$ powders, and compression loads were then applied using a customized mechanical load frame. As a result, the packing underwent confined uniaxial compression during in situ neutron diffraction. The neutron beam was centered on the middle of the packing and was collimated to have a gauge volume of $5 \times 5 \times 5 \mathrm{~mm}^{3}$. The detected neutrons thus were free from being diffracted by the rams or the die. After testing, the resultant dense compact was $\sim 10 \mathrm{~mm}$ in diameter and $\sim 15 \mathrm{~mm}$ in height. 
(a)

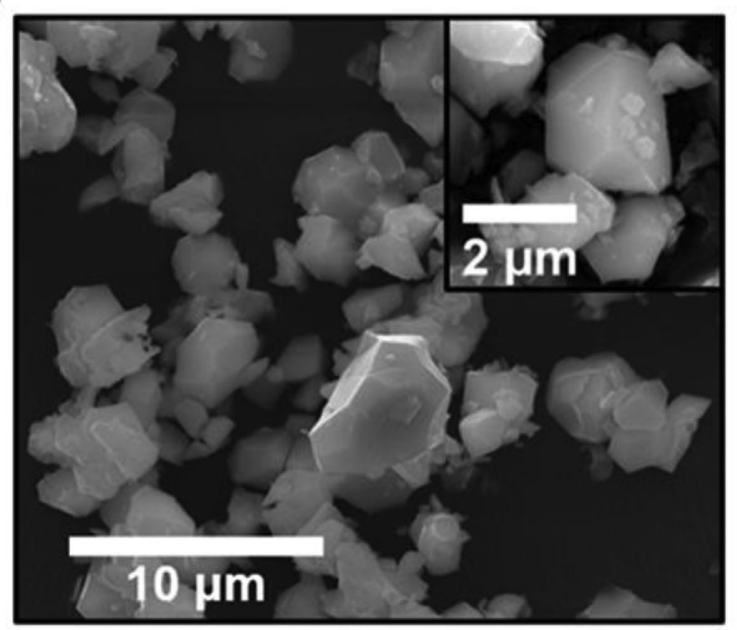

(b)

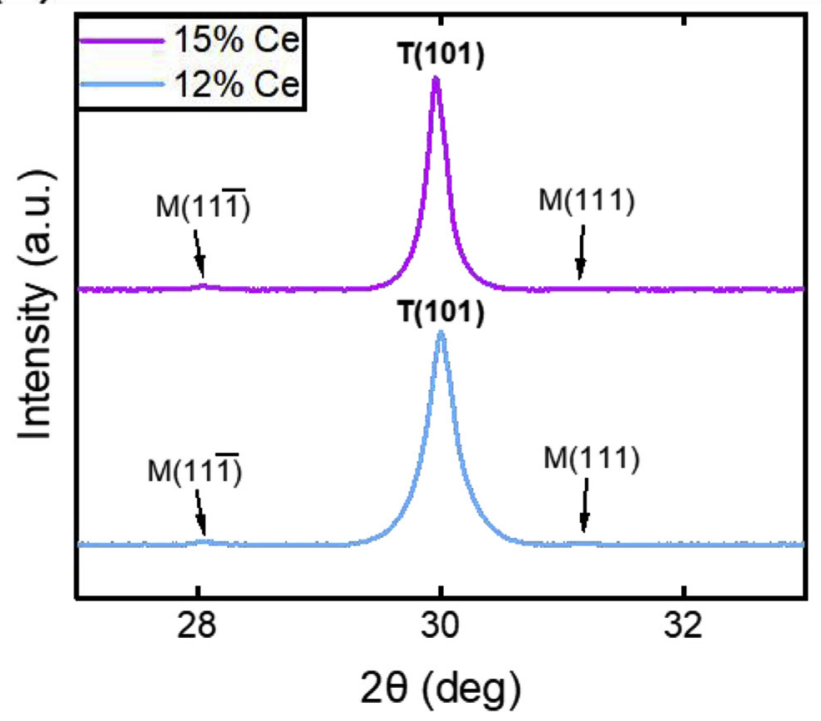

Fig. 1. (a) SEM micrograph of single crystal $\mathrm{ZrO}_{2}-\mathrm{CeO}_{2}$ particles. (b) XRD two-theta scans of the synthesized packings, where $\mathrm{T}$ denotes the tetragonal structure (austenite) and $\mathrm{M}$ denotes the monoclinic phase (martensite).

Two distinct continuous in situ neutron diffraction experiments were performed. The first was with the $\mathrm{ZrO}_{2}-12$ at\% $\mathrm{CeO}_{2}$ packing, involving mechanical loading, unloading, and subsequent heating. The particles were placed into a steel sleeve before being slightly pre-compressed to $2 \mathrm{kN}$ (i.e. an average axial stress of $25 \mathrm{MPa}$ ). The steel sleeve was used here because of its high-temperature yield strength, which was necessary for the in situ heating experiment. The compression load was increased quasi-statically up to a maximum load of $78.5 \mathrm{kN}$ (i.e. an average axial stress of $1 \mathrm{GPa}$ ). After that, the packing was unloaded quasi-statically until it reached zero axial load. The packing was then heated under loadcontrol mode using an induction coil. This raised the temperature from room temperature to a maximum of $513 \mathrm{~K}$. At each temperature step of $50 \mathrm{~K}$, the packing was allowed to equilibrate for $30 \mathrm{~min}$.

The second experiment was with the $\mathrm{ZrO}_{2}-15$ at $\% \mathrm{CeO}_{2}$ packing, involving mechanical loading and unloading under quasi-static conditions. This powder was confined by an aluminum sleeve, which was both neutron transparent and sufficiently strong at room temperature. Bulk $\mathrm{ZrO}_{2}-15$ at\% $\mathrm{CeO}_{2}$ is known to exhibit superelasticity, but whether reversible SIMT can occur in the granular form was unknown before this study. To facilitate reversible SIMT in granular packings, we pre-compressed the powders with a relatively high load of $78.5 \mathrm{kN}$ and then unloaded it to $2 \mathrm{kN}$ before in situ experiments. This made the packing more compact without significant particle rearrangement or fracture events during subsequent loading and data collection. For all mechanical measurements, the load-displacement curve of the packing was obtained by subtracting the deformation of empty die, as detailed elsewhere [37].

\subsection{Processing of neutron diffraction data}

After both tests, the continuously collected data was reduced using VDRIVE [38]. This separated the continuous diffraction data into distinct time increments through a binning process. Bin widths of $30 \mathrm{~min}$ for the $\mathrm{ZrO}_{2}-12$ at\% $\mathrm{CeO}_{2}$ packing and $10 \mathrm{~min}$ for the $\mathrm{ZrO}_{2}-$ $15 \mathrm{at} \% \mathrm{CeO}_{2}$ packing were used to reduce the level of noise and volume of data.

In both experiments, the martensite phase fraction was extracted from the diffraction pattern collected from detector bank 1 using Rietveld refinement with GSAS and EXPGUI [39-41]. Fig. 2(b) shows an example of the Rietveld refinement of the first timestep from compression of the $\mathrm{ZrO}_{2}-12$ at $\% \mathrm{CeO}_{2}$ packing. Diffraction points are blue ' $x$ ' signs, and the fitted pattern is in red. The orange line at the bottom indicates the small residual diffraction data, which is largely clustered around exceptionally broad peaks. The lattice strain and broadness of the austenitic tetragonal (101) peak from the same patterns were calculated via single-peak Gaussian profile fitting (Fig. 2(c)). This peak was used for analysis because of its lack of convolution with other peaks and its high intensity. The loading conditions in this work were not observed to lead to substantial texture formation in tetragonal phase in the packings, and the analysis of tetragonal (101) peak was considered to be representative of the general evolutionary trends. Data for the paused periods and lengthy periods of loading were averaged, with the error bars representing the standard deviation.

\section{3. $\mathrm{ZrO}_{2}-12$ at\% $\mathrm{CeO}_{2}$ packing: irreversible SIMT and thermally- induced reverse transformation}

\subsection{Phase evolution during loading, unloading, and subsequent heating}

The phase evolution in the $\mathrm{ZrO}_{2}-12$ at\% $\mathrm{CeO}_{2}$ packing is monitored during loading, unloading, and subsequent heating using neutron diffraction. Fig. 3(a) is a surface plot of the in situ neutron diffraction intensity versus d-spacing (horizontal axis) and time (vertical axis), with periods of loading and unloading indicated along the vertical axis. Within the d-spacing range shown here, there is a profound evolution of the peak intensity at $2.98 \AA$, which corresponds to tetragonal (101) peak. This figure clearly presents a decrease of the peak intensity during loading (transitioning from red to yellow) but no trend during unloading (remaining yellow). This observation confirms SIMT during loading, without transformation during unloading. Note that the observed trend is not caused by the increase of packing density during compression, which should lead to an inverse trend of increasing intensity with load.

Based on Rietveld refinement of all peaks in the neutron diffraction pattern, a quantitative plot of the phase evolution is shown in Fig. 3(b). During loading, the monoclinic phase (volume) fraction is seen to continuously increase from $6 \mathrm{vol} \%$ to $13 \mathrm{vol} \%$ as the load increases to $78.5 \mathrm{kN}$ (axial stress of $1 \mathrm{GPa}$ ). Unambiguously, 
(a)

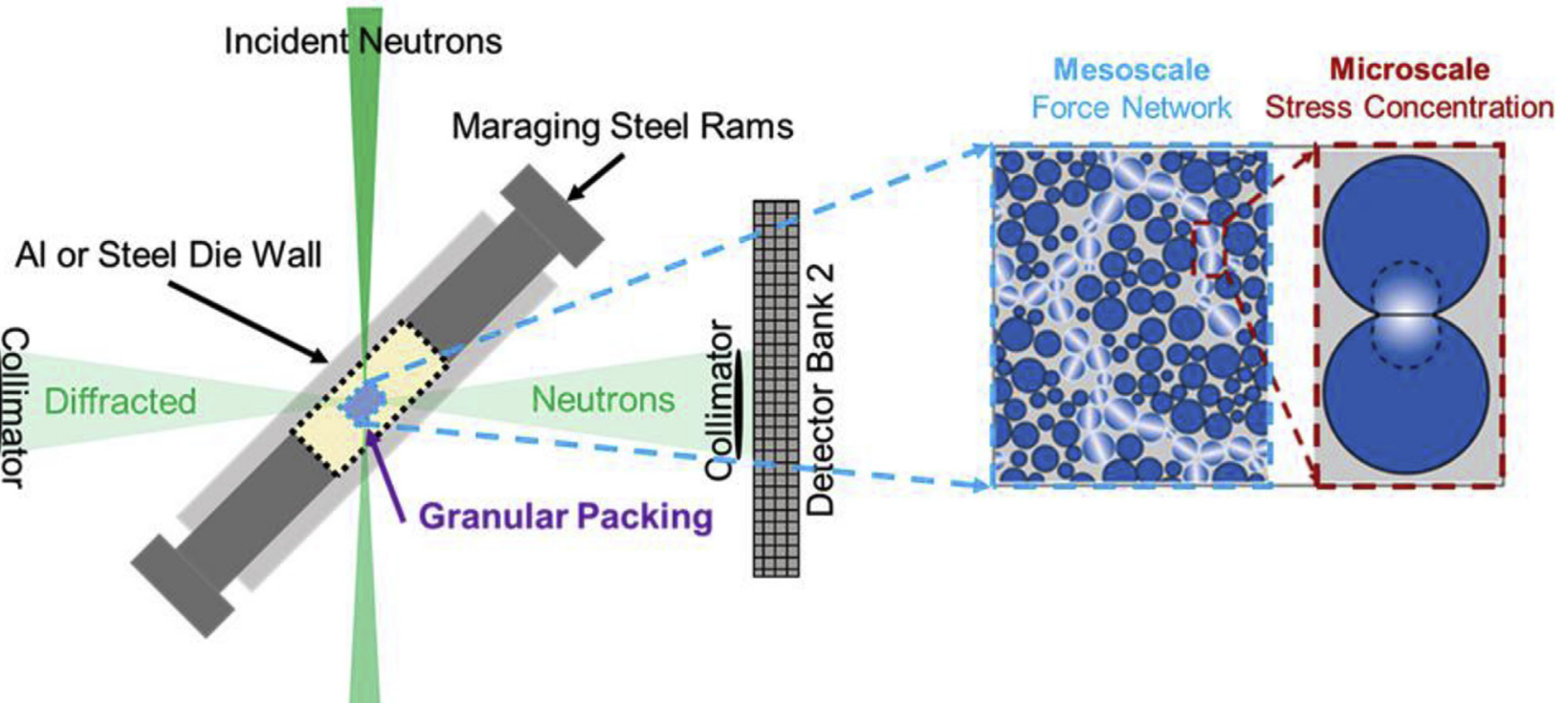

Transmitted Neutrons

(b)

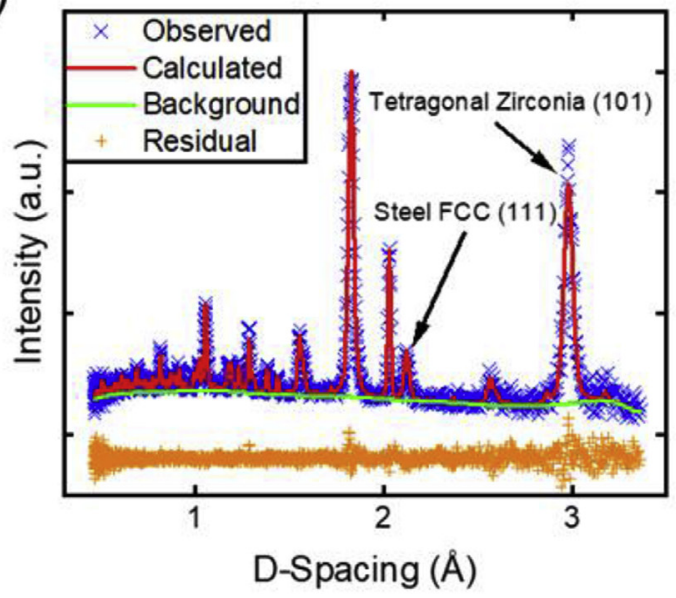

(c)

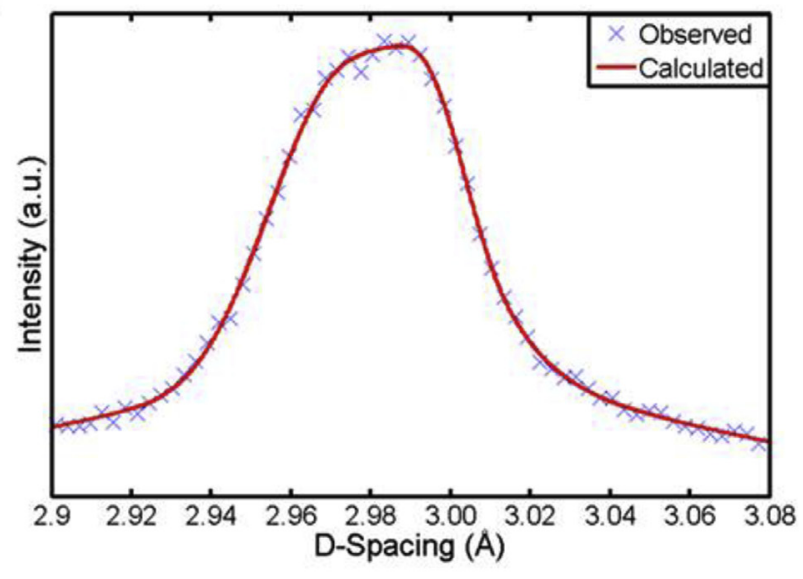

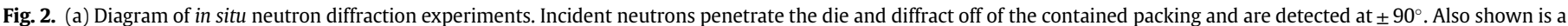

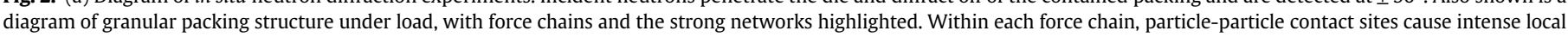

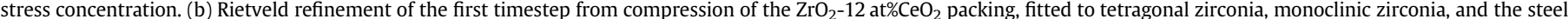

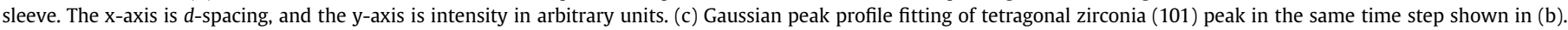

SIMT occurs across the entire range of applied loads without a visible critical load or plateau. This is contrary to the classical case of a monolithic, austenitic $\mathrm{ZrO}_{2}-\mathrm{CeO}_{2}$ that requires a critical stress (usually on the order of $\sim 1 \mathrm{GPa}$ ) to trigger the transformation, after which SIMT proceeds rapidly through autocatalytic and selfaccommodating (dubbed 'bursting') transformation modes until the driving force is no longer sufficient for transformation $[15,42]$.

The transformation behavior difference between granular packings and monolithic solids stems directly from the heterogeneous structure of the granular packing, which concentrates the applied load in a few particles at a time. This manifests as mesoscale force chains and force networks, wherein a portion of particles support all of the applied load [21,43-45]. On the microscale, the load on a force chain leads to stress concentration in the regions close to the particle contact. Overall, a small macroscopic load, which leads to very high stress concentration in some particles, can result in local martensite nucleation. Increasing the macroscopic load gradually increases the number of particles that experience critical stress, resulting in the observed continuous increase of the martensite phase fraction. Such a continuous mode of transformation has indeed been observed in previous work using ex situ phase characterization [28]. However, without in situ phase characterization, it was unclear how much of the transformed phase reversed during unloading.

The in situ neutron diffraction method in this work provides transformation information during unloading. Fig. 3 (b) shows that when the load is released, there is no substantial change in martensite phase fraction, suggesting that all of the martensite formed during compression remains. This irreversible behavior is similar to that observed in the monolithic counterpart [10]. Because the austenite phase is metastable and $T_{0}<A_{S}$, even under zero applied load the transformed particles have insufficient driving force to transform back to austenite. However, closer scrutiny of Fig. 3(b) reveals a trend of slight increase of martensite fraction during unloading. This trend, although barely discernible above the error bars, is interesting and counterintuitive-an increase of 
(a)

(b)
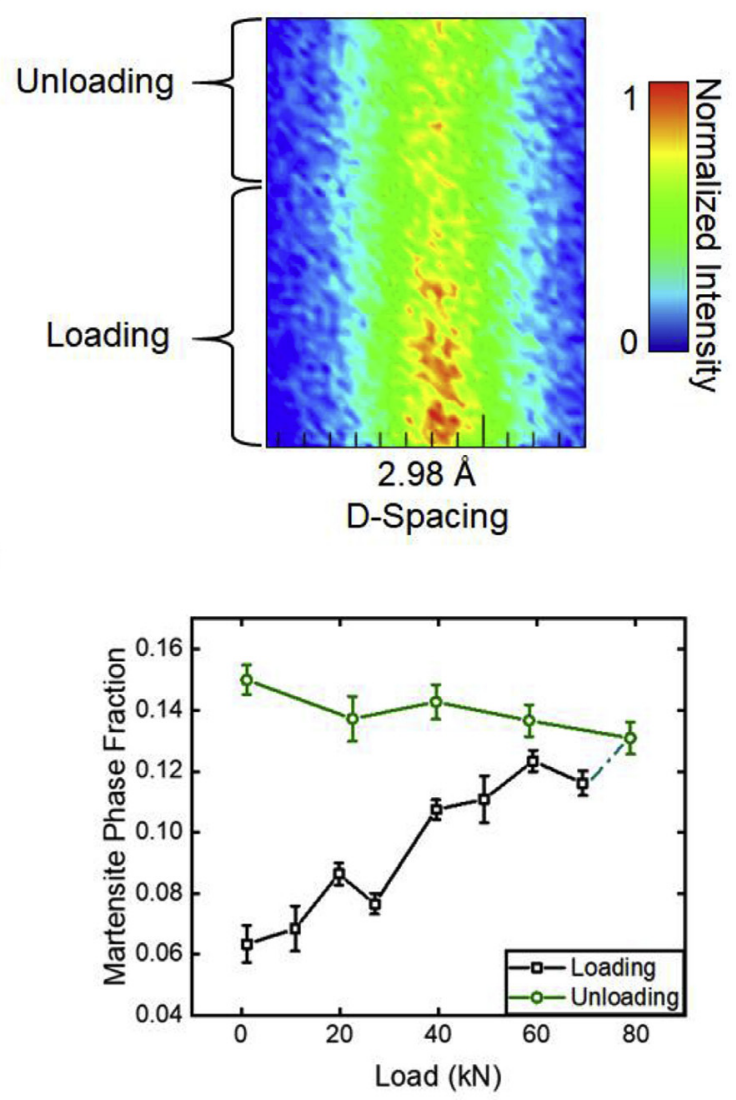

Fig. 3. (a) Evolution of the tetragonal (101) peak intensity at D-spacing of $2.98 \AA$ in the $\mathrm{ZrO}_{2}-12$ at\% $\mathrm{CeO}_{2}$ packing, with periods of increasing load (loading) and decreasing load (unloading) indicated. (b) Results of phase evolution based on Rietveld refinement for the $\mathrm{ZrO}_{2}-12$ at\% $\mathrm{CeO}_{2}$ experiment.

martensite should be a result of stress increase rather than decrease. That being said, the slight increase of martensite during unloading can be attributed to the complex granular mechanics that dominate the loading conditions at every independent time step. As observed from granular physics work such as in Refs. [46-49], decrease of the macroscopic load leads to dynamic evolution of the contact network inside the packing, and therefore force redistribution among the particles. Some individual particles that were not part of the strong network during loading can suddenly begin carrying significant force due to changes in the local packing structure. In the present experiment, this should cause them to transform to martensite during unloading. Since stressinduced martensitic transformation is irreversible at composition $\mathrm{ZrO}_{2}-12$ at\% $\mathrm{CeO}_{2}$, the already transformed volume during loading should not transform back upon unloading. Then, the additional transformed volume due to force redistribution during unloading can cause an overall, slight increase of the martensite phase.

After unloading, the packing is in a jammed compact state with zero average stress. During subsequent heating, the reverse transformation is observed by neutron diffraction. Fig. 4 plots the phase evolution as the temperature increases from $300 \mathrm{~K}$ to $513 \mathrm{~K}$. From $300 \mathrm{~K}$ to $400 \mathrm{~K}$, the martensite phase fraction shows only a slight decrease from the as-unloaded value. As the temperature is further increased, however, the martensite phase fraction substantially decreases.

The initial reluctance of the reverse transformation indicates that the lowest possible $A_{S}$ temperature for the reverse

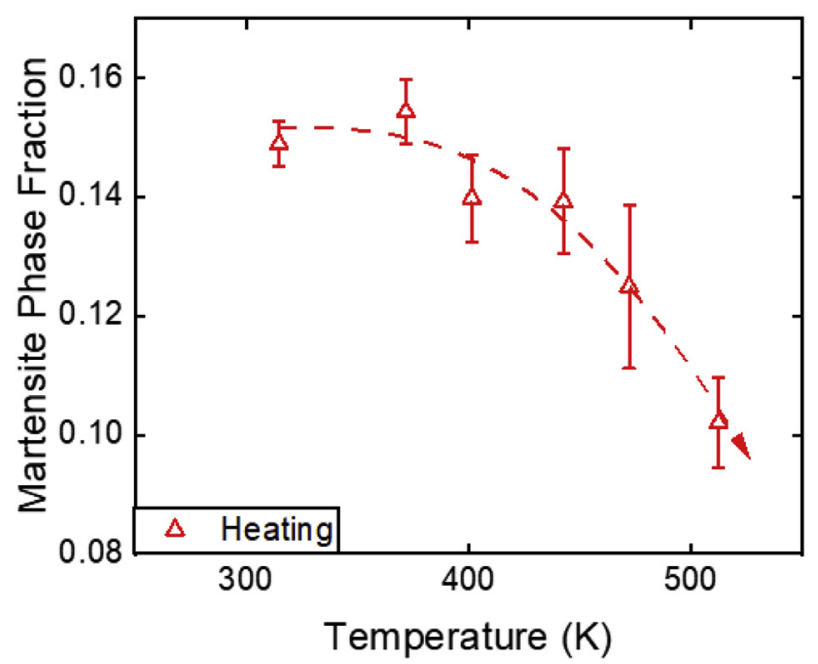

Fig. 4. Thermally-induced reverse transformation of $\mathrm{ZrO}_{2}-12$ at $\% \mathrm{CeO}_{2}$ packing after loading and unloading. After heating to the maximum temperature of $513 \mathrm{~K}$, the packing still retains $\sim 10 \%$ martensite.

transformation is $\sim 400 \mathrm{~K}$. However, even when heating to $513 \mathrm{~K}$, the original phase fraction was still not fully recovered, with $10 \mathrm{vol} \%$ martensite remaining. That is to say, from $400 \mathrm{~K}$ to $513 \mathrm{~K}$, the martensite phase fraction only decreases from $14 \mathrm{vol} \%$ to $10 \mathrm{vol} \%$, indicating that for some particles the $\mathrm{A}_{\mathrm{F}}$ temperature has not been reached. This broad temperature window for reverse transformation is in striking contrast with the monolithic case, which shows $100 \%$ transformation between $A_{S}$ and $A_{F}$ that are usually separated by less than $50 \mathrm{~K}[50]$.

The observed wide transformation temperature window can be attributed to stress heterogeneity in the packing; during compression, each particle experiences different levels of stress so that the transformed volume fraction can be vastly different among individual particles. Because of the lack of cohesion in a granular packing, the subsequent thermally-induced martensitic transformation occurs independently in individual particles, but at different temperatures. For some particles $A_{S}$ is as low as $400 \mathrm{~K}$, and for some $A_{F}$ is above $513 \mathrm{~K}$. As a result, after heating up to $513 \mathrm{~K}$, there is still $10 \%$ residual martensite. However, if we keep increasing the temperature above $513 \mathrm{~K}$, the martensite phase volume fraction should continue to decrease. We note that a thermal gradient in the granular packing due to poor thermal conductivity might also contribute to the observed wide transformation window. That being said, it is not likely to be the main reason because the packing was allowed to equilibrate for $30 \mathrm{~min}$ at each temperature step of $50 \mathrm{~K}$ during in situ heating and the thermal gradient should have been effectively reduced.

\subsection{Granular packing structure evolution}

In addition to phase evolution, in situ neutron diffraction provides the evolution of the lattice strain. The average lattice strain for a given crystal plane can be calculated by comparing the lattice spacing during and before in situ testing. By examining together the lattice strain evolution and packing microstructure evolution (from the load-displacement curve), new insights into granular structure evolution can be revealed. Fig. 5(a) and (b) compare the loaddisplacement curve with the lattice strain-displacement curve of the $\mathrm{ZrO}_{2}-12$ at $\% \mathrm{CeO}_{2}$ packing. The latter is based on the lattice strain of the tetragonal (101) plane, which is expected to be representative of the general trend, given the random texture of the tetragonal phase. 
(a)

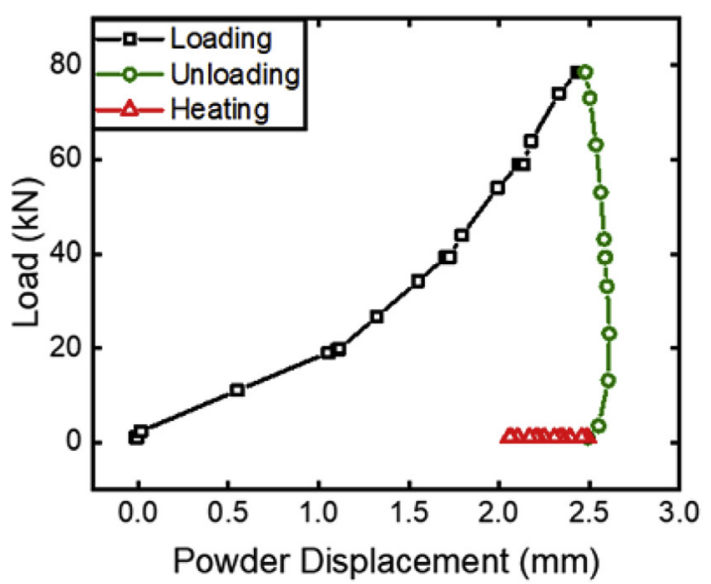

(b)

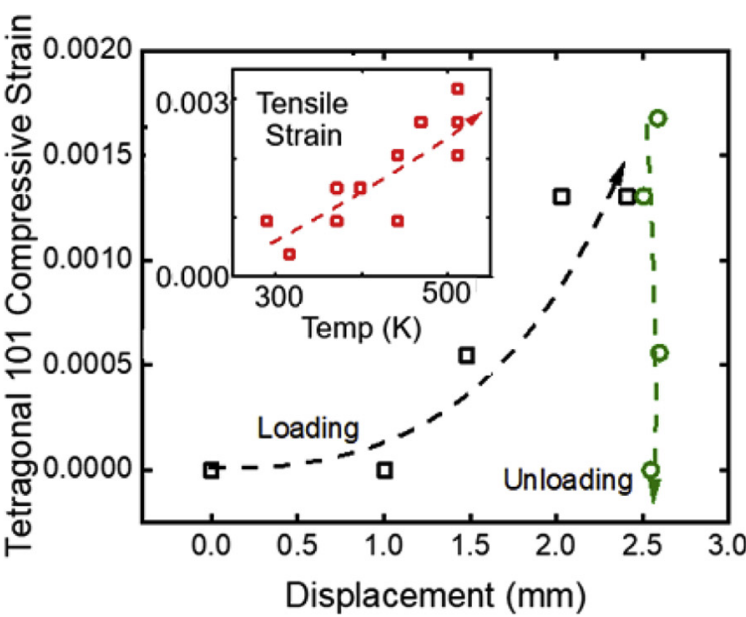

(c)

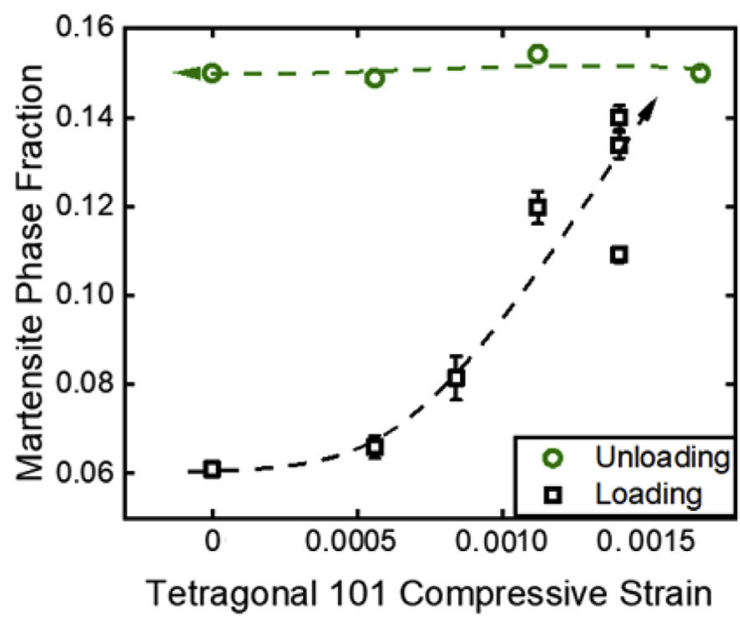

Fig. 5. (a) Macroscale mechanical response of the $\mathrm{ZrO}_{2}-12$ at $\% \mathrm{CeO}_{2}$ packing during loading, unloading, and heating. (b) Evolution of tetragonal (101) compressive strain during loading and unloading. Inset: tensile strain evolution of the tetragonal (101) peak caused by thermal expansion. (c) Martensite phase plotted against the tetragonal (101) compressive strain during loading and unloading.
During loading, displacement increases non-linearly (Fig. 5(a)) with extensive compaction taking place at low loads. Despite the rapid compaction at low loads, the average lattice strain is still almost zero until the packing reaches $\sim 1.0 \mathrm{~mm}$ of displacement (Fig. 5(b)). This implies that the structure of the packing is constantly changing to accommodate this large initial displacement, mostly as a series of rearrangements by particle slipping rather than by contact deformation. At higher loads where displacement increases more slowly, the average lattice strain climbs faster, eventually reaching a value of almost $0.2 \%$. Clearly, the initial $1.0 \mathrm{~mm}$ displacement corresponds to Stage I of powder compaction with particle rearrangement, which is followed by Stage II with the packing density increasing through contact deformation [51,52]. After the experiment, the packing density was estimated to be $\sim 70 \%$ of the theoretical density for zirconia. This is typical for a granular ceramic with a monodisperse particle size distribution.

During unloading, the displacement is almost static, whereas the average lattice strain decreases and eventually returns to zero. This shows that the packing structure has reached a stable state, as is reported in studies of dense granular media [53]. As the packing is then heated under load control (maintained at $0 \mathrm{kN}$ ), the packing expands axially by almost $0.5 \mathrm{~mm}$ (red dots in Fig. 5(a)). This corresponds to $\sim 3.3 \%$ dilation, by considering that the resultant packing would be $15 \mathrm{~mm}$ tall without heating. The inset of Fig. 5 (b) presents the lattice strain evolution of the tetragonal (101) peak, showing a $0.3 \%$ increase of lattice constant over a temperature range of $240 \mathrm{~K}$. This lattice strain is consistent with what would be caused by thermal expansion, because the coefficient of thermal expansion of zirconia is known to be on the order of $10^{-5} / \mathrm{K}$ [54]. The reverse phase transformation from martensite to austenite in zirconia involves a $\sim 5 \%$ volume shrinkage. At the end of the heating experiment, the observed martensite phase fraction is seen to decrease by $4 \%$ (from $14 \%$ to $10 \%$ ), so the reverse transformation can directly account for $0.2 \%$ volumetric contraction, or $-0.2 \%$ volumetric strain. Assuming isotropic contraction, this would lead to a linear contraction of $0.067 \%$. The measured 3.3\% linear dilation, which may be viewed as the macroscopic recovery strain, apparently cannot be accounted for just by the thermal expansion $(0.3 \%$ strain) or thermally-induced reverse martensitic transformation ( $-0.067 \%$ strain). The unaccounted $3.067 \%$ could be attributed to the particle rearrangement and structure evolution of the packing. This analysis suggests that the packing expansion observed in Fig. 5 (a) is mainly caused by packing structure evolution rather than thermal expansion or reverse martensitic transformation.

Finally, by comparing Fig. 5 (b) and Fig. 3 (b), we plot the phase vs. lattice strain curve in Fig. 5 (c). There is almost no SIMT found up to $0.05 \%$ elastic strain of the tetragonal (101) plane, after which the martensite phase fraction increases consistently with the lattice strain. This behavior suggests that within Stage II of powder compaction by contact deformation, SIMT occurs only after the average contact deformation is large enough (after 0.05\% (101) strain).

From the SIMT perspective, we conclude that multiple stages are involved in granular compression and illustrate this in Fig. 6. These stages are characterized by 1 ) particle rearrangement (with 0-1.0 mm displacement and zero tetragonal (101) lattice strain), 2) contact deformation (with 1.0-1.5 mm displacement and 0-0.05\% tetragonal (101) lattice strain), and 3) simultaneous contact deformation and martensitic transformation (beyond 0.05\% tetragonal (101) lattice strain), respectively.

\section{4. $\mathrm{ZrO}_{2}-15 \% \mathrm{CeO}_{2}$ packing: direct evidence of reversible SIMT}

The phase evolution in the $\mathrm{ZrO}_{2}-15$ at $\% \mathrm{CeO}_{2}$ packing is 


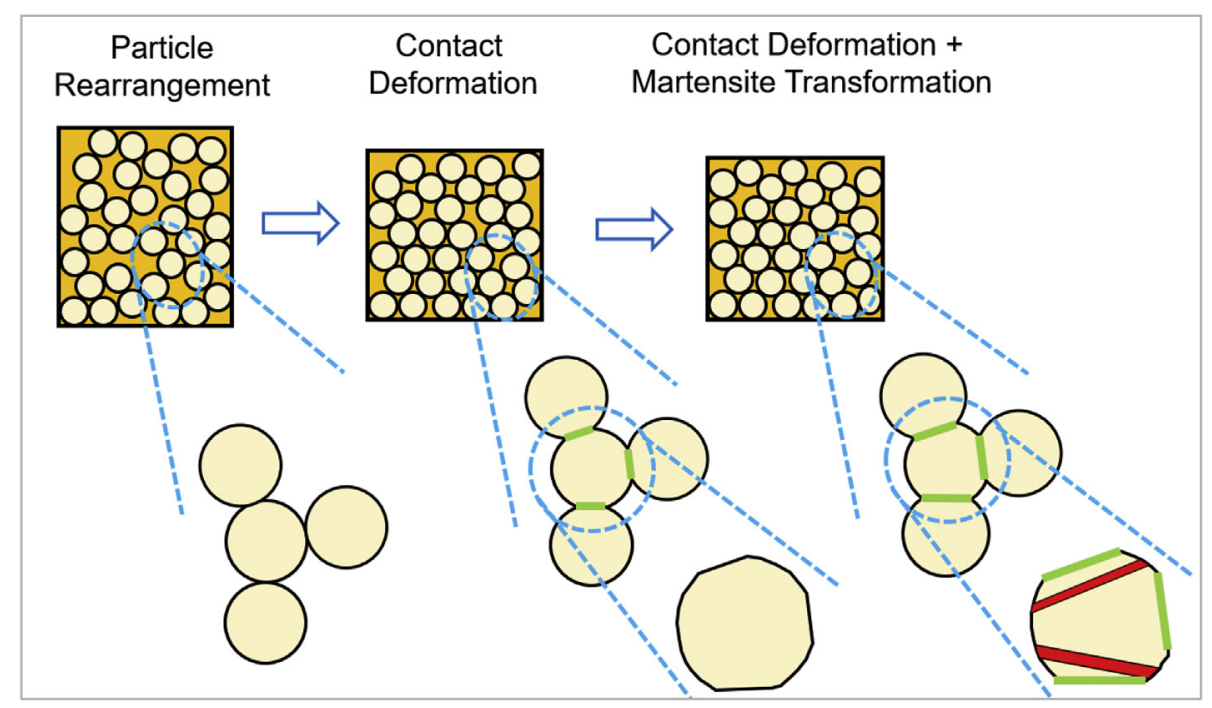

Fig. 6. Illustration of the co-evolution of packing microstructure, lattice strain, and phase during compression.

monitored during loading and unloading. Fig. 7 shows the martensite phase fraction resulting from Rietveld refinement of the neutron diffraction patterns. Recall that, at this composition, both $A_{S}$ and $A_{F}$ fall below room temperature. Loading and unloading are seen to lead to forward and reverse martensitic transformation, respectively, displaying a hysteresis that reflects the energy absorptive nature of martensitic transformation. This provides the first direct evidence that reversible, stress-induced martensitic transformation can occur in granular materials even without bursting effects [42], relying solely on individual nucleation events. Upon complete unloading, the original stress-free phase fraction is fully recovered.

During loading, the maximum observed martensite phase fraction of 11.8 vol\% is reached at the maximum load of $78.5 \mathrm{kN}$. This shows that even with an average axial stress of $1 \mathrm{GPa}$, the amount of transformation in the packing is only $2.3 \%$ (increase from the initial 9.5\%). Such a low transformation volume is mainly a result of the highly heterogeneous stress distribution in a granular packing. Microscopic stress concentration inside individual particles only allows for partial transformation, leaving some of the

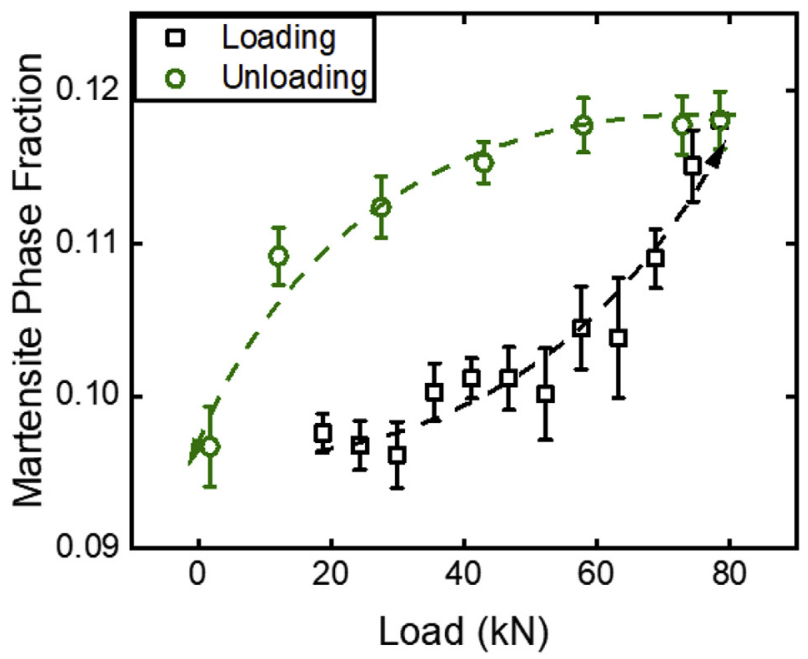

Fig. 7. Phase evolution of the $\mathrm{ZrO}_{2}-15$ at $\% \mathrm{CeO}_{2}$ packing during loading and unloading showing complete reversibility. volume still in the austenite phase; mesoscopic force networks preclude many particles from bearing high load at all, leaving them entirely austenitic. There is also a macroscopic stress heterogeneity in the packing in the in situ neutron diffraction experiment: the load-bearing particles near the rams effectively shield a large number of the inner particles from load. To avoid interfering signals from the rams and die sleeve, the neutron beam is restricted to particles at the very center of the packing, which is $\sim 7.5 \mathrm{~mm}$ away from each ram. With the shielding from load-bearing particles near the ram, there is generally a low probability of these particles bearing high enough load to achieve critical stress and undergo the martensitic transformation. Furthermore, the number of $1 \mathrm{GPa}$ is not very high for stress-induced martensitic transformation in $\mathrm{ZrO}_{2}-15 \% \mathrm{CeO}_{2}$. In fact, from micropillar testing, the critical stress for transformation in such (or similar) composition is estimated to be on the order of $1 \mathrm{GPa}$ [14]. Convolution of the intrinsically high critical stress with the stress heterogeneities on the macro-, meso-, and microscale leads to the low transformation volume in the $\mathrm{ZrO}_{2}-$ $15 \% \mathrm{CeO}_{2}$ packing observed in Fig. 7.

The slope of the plot in Fig. 7, which is a measure of the transformation rate with respect to axial load, shows different evolution behavior during loading and unloading. During loading, a substantial increase of the transformation rate is seen once the load reaches $55 \mathrm{kN}$ (i.e. average axial stress of $700 \mathrm{MPa}$ ). During unloading, there is minimal reversion to austenite until the load drops below $20 \mathrm{kN}$ (i.e. average axial stress of $255 \mathrm{MPa}$ ). After that, reverse transformation occurs rapidly with load removal and the packing eventually returns to its initial martensite phase fraction. Overall, for the same external load, the martensite phase fraction is higher during unloading than loading. This mainly originates from the difference of critical stress for forward and reverse transformation. In superelasticity, the critical stress for forward transformation is higher than that for reverse transformation [2]. This means that any volume of material originally experiencing an equivalent stress greater than the forward transformation stress can stay in the martensite phase until its stress level drops below the reverse transformation stress. That is, with the stress level ranging between the reverse transformation stress and forward transformation stress, the material is in the tetragonal phase during loading but is in the monoclinic phase during unloading. As a result, even with the similar stress distribution, there is more monoclinic phase during unloading than during loading. Ultimately, the 
difference in critical stress for forward and reverse transformation manifests as a hysteresis loop in stress-strain or stresstransformation volume plots for all such materials as shown in Fig. 7.

In principle, irreversible packing structure evolution could be another cause of the different transformation rates during loading and unloading. However, this is not the case of $\mathrm{ZrO}_{2}-15$ at $\% \mathrm{CeO}_{2}$ packing in Fig. 7, because the packing was pre-compressed at a high load and reached a relatively stable microstructure before in situ neutron diffraction. This conclusion is supported by Fig. 8, which shows the lattice strain evolution of the $\mathrm{ZrO}_{2}-15$ at $\% \mathrm{CeO}_{2}$ packing. The lattice strain for the same load is seen to be similar during loading and unloading, while the displacement is almost completely recovered. Therefore, the same magnitude of load during loading and unloading corresponds to a similar packing structure and stress distribution.

One may also argue that the different transformation rates in loading and unloading could be a result of friction between the die wall and the powders. However, because the neutron beam is collimated to a $5 \times 5 \times 5 \mathrm{~mm}^{3}$ volume and the packing is $10 \mathrm{~mm}$ in diameter, such edge effects are unlikely to dominate the behavior in the region of observation.

\section{Evolution of lattice strain and peak broadness in both packings}

\subsection{Reversible lattice strain evolution}

As shown in Figs. 5(b) and Figure 8, the tetragonal (101) lattice strain does behave elastically in both $\mathrm{ZrO}_{2}-12$ at\% $\mathrm{CeO}_{2}$ and $\mathrm{ZrO}_{2}-$ 15 at\% $\mathrm{CeO}_{2}$ packings, with a decrease of the applied load directly leading to a decrease in the lattice strain. Upon full unloading, both packings reach a zero macroscopic strain state, although microscopic stress and strain may still exist. At the same load, the magnitude of lattice strain is different in the two experiments due to differences in their preparation and initial packing density, as detailed in Sections 2, 3, and 4. The $\mathrm{ZrO}_{2}-15$ at $\% \mathrm{CeO}_{2}$ packing was pre-compressed at a higher load before in situ testing, and thus had a higher packing density and a higher lattice strain level for a given load.

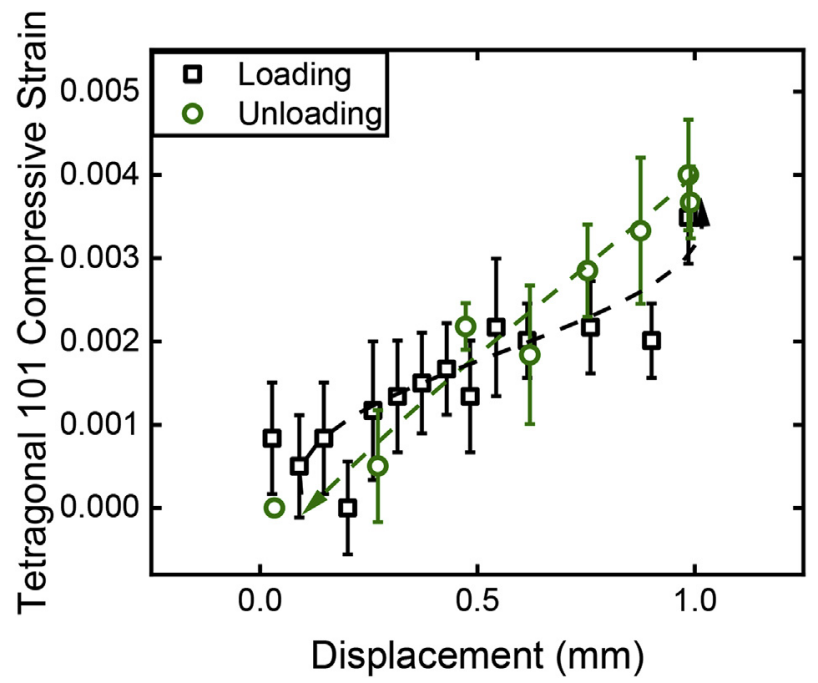

Fig. 8. Evolution of the tetragonal (101) compressive strain in the $\mathrm{ZrO}_{2}-15$ at $\% \mathrm{CeO}_{2}$ packing, which is plotted against displacement.

\subsection{Correlation of the peak broadness and phase transformation}

In our collected data, the neutron diffraction signal is plotted as a function of $d$-spacing. We thus define the integral broadness of a peak as the ratio of the area under the peak to its maximum intensity: $B=\left(\int_{d_{\text {Left }}}^{d_{\text {Right }}} I \times \delta d\right) / I_{\text {Max }}$, where $d_{\text {Left }}$ and $d_{\text {Right }}$ are the $d$ spacing at the two edges of the peak, and $I_{\text {Max }}$ is the maximum peak intensity. By this definition, the integral peak broadness has a unit of $\AA$ and measures the range of $d$-spacings that contribute substantially to the diffraction peak.

In Fig. 9(a) and (b), we measure the broadness of the tetragonal (101) diffraction peak and plot its evolution during loading and unloading of $\mathrm{ZrO}_{2}-12$ at\% $\mathrm{CeO}_{2}$ and $\mathrm{ZrO}_{2}-15$ at\% $\mathrm{CeO}_{2}$ packings, respectively. This peak is selected because it corresponds to the majority phase in both experiments, has high intensity, is not convoluted with any adjacent peaks, and its shape is readily extracted. In the case of irreversible SIMT $\left(\mathrm{ZrO}_{2}-12 \mathrm{at} \% \mathrm{CeO}_{2}\right)$, the integral broadness increases by nearly $12 \%$ (from $~ 0.026$ to $\sim 0.029 \AA$ ) during loading. The peak broadness remains almost constant during unloading (Fig. 9(a)), although the lattice strain is

(a)

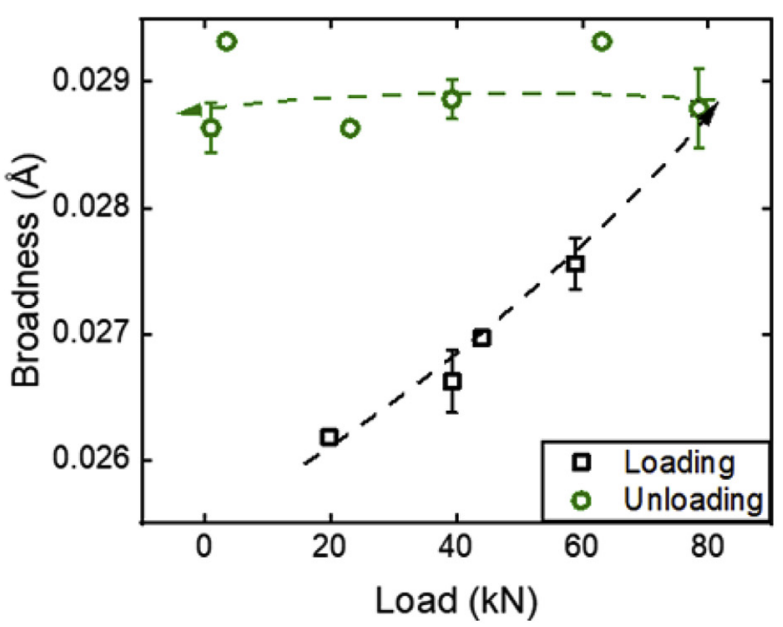

(b)

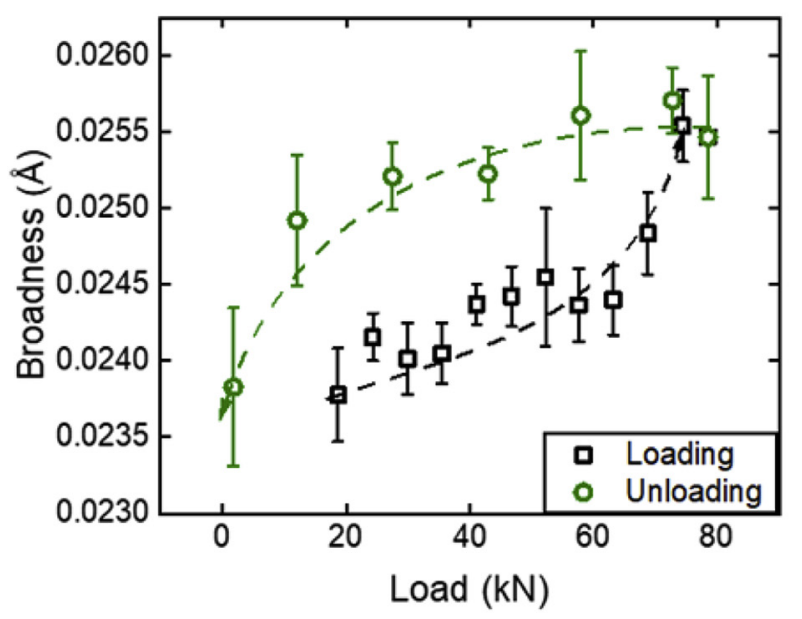

Fig. 9. Peak broadness evolution during loading and unloading in (a) the $\mathrm{ZrO}_{2}-12$ at\% $\mathrm{CeO}_{2}$ and (b) the $\mathrm{ZrO}_{2}-15$ at\% $\mathrm{CeO}_{2}$ packing. Evolution of the peak broadness is irreversible in (a), and is reversible in (b). 
completely relaxed. In the case of reversible SIMT $\left(\mathrm{ZrO}_{2}-15\right.$ at\% $\mathrm{CeO}_{2}$ ), a similar increase in the broadness of 9\% (from $\sim 0.0235$ to $\sim 0.0255 \AA$ ) is observed during loading, but it returns almost completely to its initial value upon unloading (Fig. 9 (b)).

A change in the integral broadness can signify several phenomena, typically plastic deformation via dislocation accumulation, grain size or particle size reduction due to fracture, or microscopic strain generation by some specific mechanisms. Plastic deformation, while observed in microscale zirconia [12], is irreversible and cannot explain the reversible evolution of peak broadness observed in the $\mathrm{ZrO}_{2}-15 \% \mathrm{CeO}_{2}$ packing. Therefore, it can be ruled out as the principal mechanism at work. Fracture is inevitable during loading, leading to reduction of the particle size and diffracting domains. While this could lead to peak broadening during loading, it again fails to explain the reversible change of broadness observed in the $\mathrm{ZrO}_{2}-15$ at $\% \mathrm{CeO}_{2}$ packing. It is also unlikely that the broadness is caused by the contact force distribution, which would require the broadening to be reversible. Although this is true for the $\mathrm{ZrO}_{2}-15$ at\% $\mathrm{CeO}_{2}$ packing in Fig. 9(b), it fails to explain the remaining broadness upon full unloading of the $\mathrm{ZrO}_{2}-12$ at\% $\mathrm{CeO}_{2}$ packing in Fig. 9(a). In addition to these unlikely mechanisms for peak broadening, other phenomena specific to tetragonal zirconia such as ferroelastic switching can be discounted by comparing the corresponding peak broadness evolution reported in literature to the observation here $[55,56]$. By process of elimination, the broadness change is likely to signify a heterogeneous micro-strain distribution in individual particles.

It is interesting that the peak broadening evolution is irreversible in the $\mathrm{ZrO}_{2}-12 \mathrm{at} \% \mathrm{CeO}_{2}$ packing, which shows irreversible SIMT, and is reversible in the $\mathrm{ZrO}_{2}-15$ at\% $\mathrm{CeO}_{2}$ packing, which shows reversible SIMT. In fact, the peak broadening trends almost perfectly mirror the phase transformation behavior observed in Sections 3 and 4. As shown in Fig. 10(a) and (b), the peak broadness follows the same trend with respect to martensite phase fraction during forward transformation in both systems, as well as in reverse transformation of the $\mathrm{ZrO}_{2}-15$ at $\% \mathrm{CeO}_{2}$ packing. During unloading of $\mathrm{ZrO}_{2}-12$ at $\% \mathrm{CeO}_{2}$, no transformation occurs, and there is no apparent evolution of the peak broadness (circled in Fig. 10 (a)). These results suggest that there is possibly a micro-strain distribution in the granular packings, which is closely related to forward and reverse SIMT.

One possible explanation of this strong correlation is the existence of a micro-strain distribution associated with each martensite-austenite interface in individual transforming particles. Since martensite growth is athermal, the glissile martensiteaustenite interface is thought to consist of an array of parallel dislocations [57-59], which must lead to micro-strain distributions around the interface. As martensite plates form during loading and are eliminated during unloading, the interface dislocations and the associated micro-strain distributions would be correspondingly generated and erased. To determine whether this can be a plausible mechanism requires an understanding of the martensite nucleation and growth mechanism in SIMT of granular packings, which will be the focus next.

\section{Discussion}

To understand the observed SIMT characteristics, in this section we discuss the mechanism of martensite nucleation and growth in granular packings. By considering the fundamental differences in driving force and nucleation barrier between granular packings and monolithic solids, we show that SIMT in granular packings is nucleation-controlled and the martensite fraction increases mainly by nucleating new martensite plates in individual particles. From that, we are able to explain the experimental observations in (a)

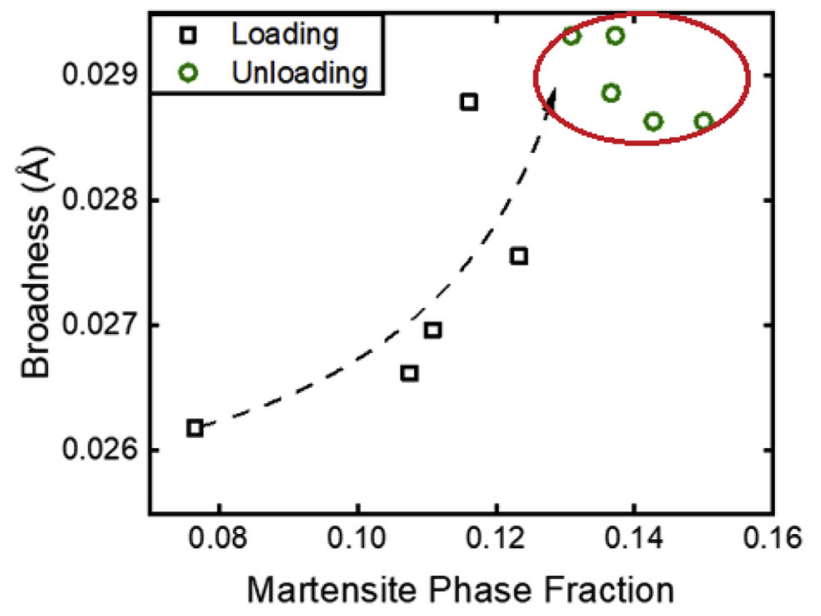

(b)

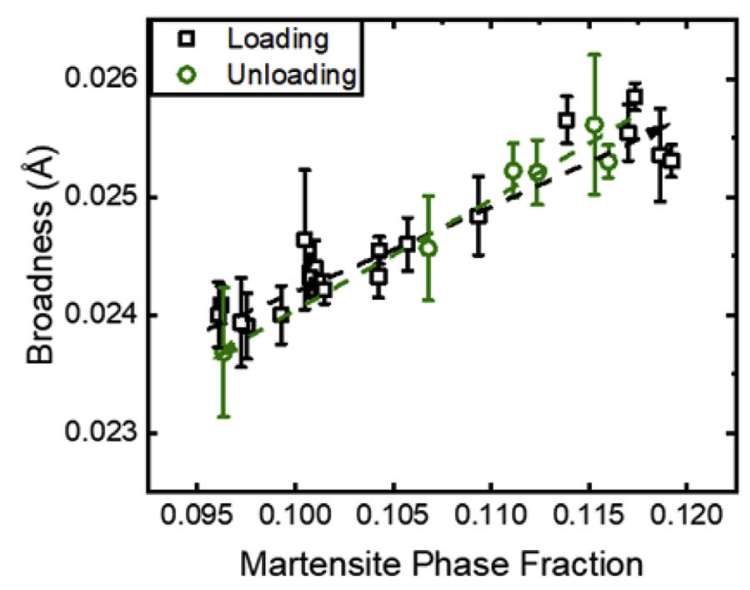

Fig. 10. Broadness trends with phase fraction during loading and unloading for (a) the $\mathrm{ZrO}_{2}-12$ at $\% \mathrm{CeO}_{2}$ and (b) the $\mathrm{ZrO}_{2}-15$ at $\% \mathrm{CeO}_{2}$ packing.

Sections 3, 4, and 5 .

\subsection{Formation of martensite plates in individual particles}

There is still debate on the mechanism of martensite nucleation [7], regarding whether it is best described by classical heterogeneous nucleation theory or the non-classical, localized soft mode (LSM) theory [60,61]. However, both theories predict that the martensite nucleation is favored in regions with free surface and local stress concentration, as detailed in Refs. [7,60-62]. Compared to a monolithic solid, a granular packing is characterized by a higher surface area-to-volume ratio and higher, localized stress concentration, which should significantly ease the martensite nucleation process. The stress distribution in a granular packing is heterogeneous on both mesoscale and microscale, so appreciable stress concentration can arise close to the inter-particle contact surface even at a low macroscopic load. The new martensite phase is thus most likely to nucleate close to such contact area with access to the free surface.

The angle between the nucleated martensite plate and the particle contact normal depends on both the stress state in the entire particle and the particle's crystallographic orientation, which restricts the available transformation orientations and variants. 
Within the context of the phenomenological theory of martensitic transformation (PTMT) in $\mathrm{ZrO}_{2}-\mathrm{CeO}_{2}$ [15], the orientation relationships (ORs) are classified and defined by the relation between the tetragonal $\mathrm{C}$-axis and the formed monoclinic axes. B-ORs

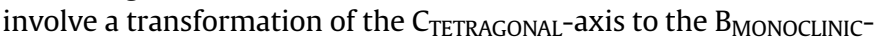
axis. C-ORs involve a direct transformation of $\mathrm{C}_{\text {TETRAGONAL-axis to }}$

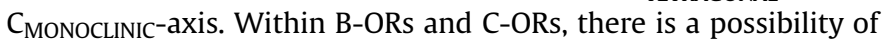
rotation that necessitates the further breakdown to B1, B2, C1, and C2 ORs, each of which has its own habit plane and shape strain direction. The habit plane and corresponding shape strain direction for each of the four ORs are shown in Fig. 11(a)-(d), which are superimposed in Fig. 11 (e). The reported habit planes are not integer crystallographic planes, and the shape strain directions are not integer vectors, but are rather computed non-integer solutions. Here, to ease comparison and visualization, we index the habit planes as their closest integer plane approximation. These drawings are made by VESTA [63].

Fig. 12 shows a schematic extrapolation of these ORs in a single- crystal tetragonal particle, with the hatch lines indicating (001) plane. For this generic particle cross-section with multiple contact sites, each contact site may nucleate a martensite variant corresponding to one of the four ORs presented in Fig. 11. Depending on the exact particle geometry and stress concentration, the preferred martensite variant should be the one that most relieves the local strain energy. This requires strong coupling of the local stress tensor and shape strain vector of the preferred variant, with their product being maximum among all possible variants. Because the martensite plates form based on localized stress concentrations and act to shield other regions from stress, they are likely to nucleate independently at each contact site. As a result, the number of martensite plates inside each particle should depend on the contact coordination number, $\mathrm{Z}$.

Once nucleated, the martensite will rapidly grow until the martensite/austenite interface encounters the free surface of the particle. While in monolithic solids long-range martensite growth is possible through autocatalytic transformation [42], in granular (a)

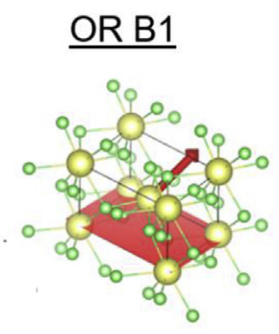

(c)

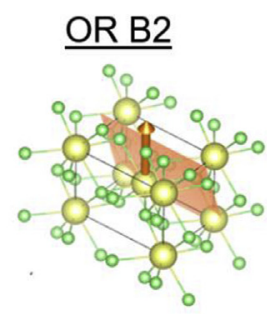

(b)

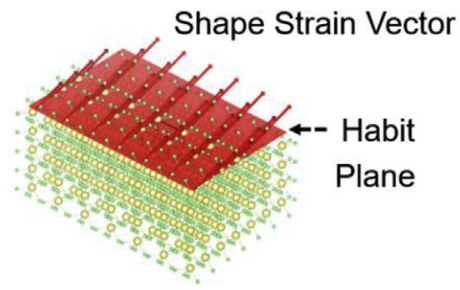

(d)

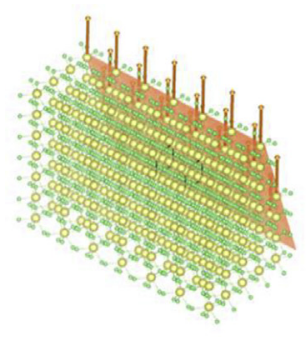

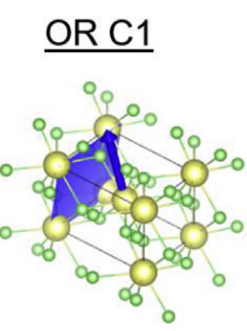

\section{$\underline{\mathrm{OR} C 2}$}
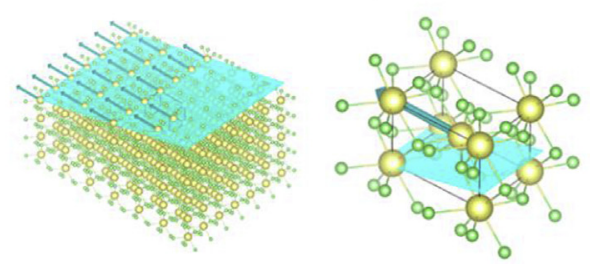

(e)

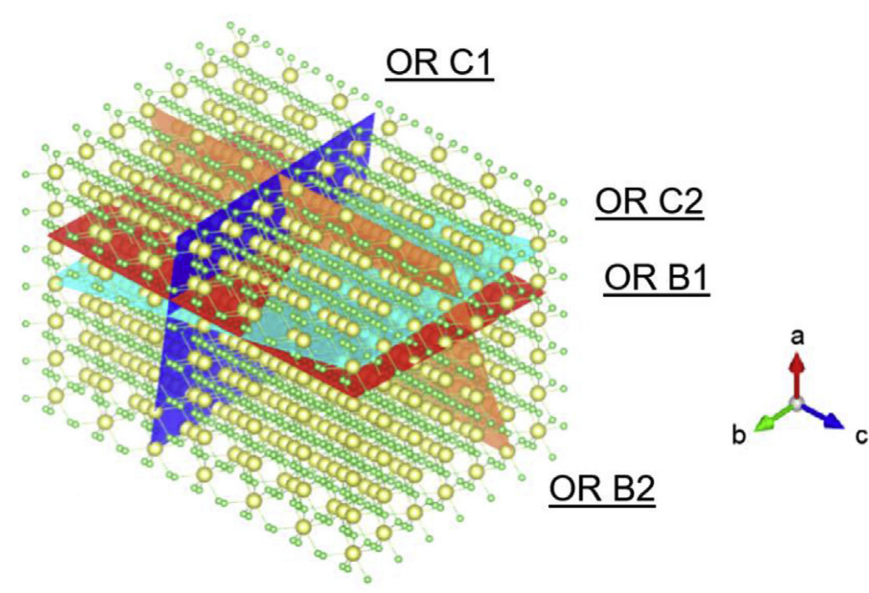

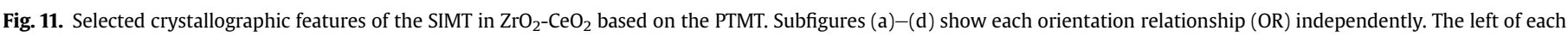

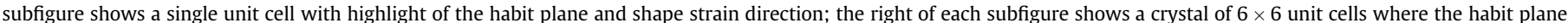

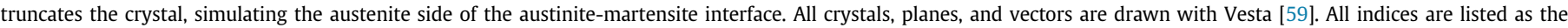

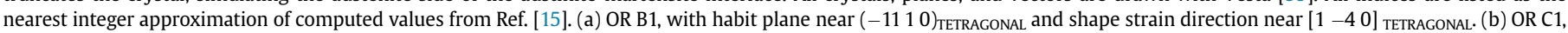

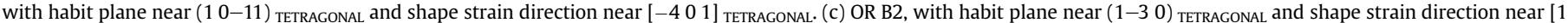

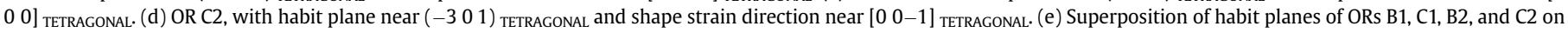
a tetragonal crystal with $6 \times 6$ unit cells. 

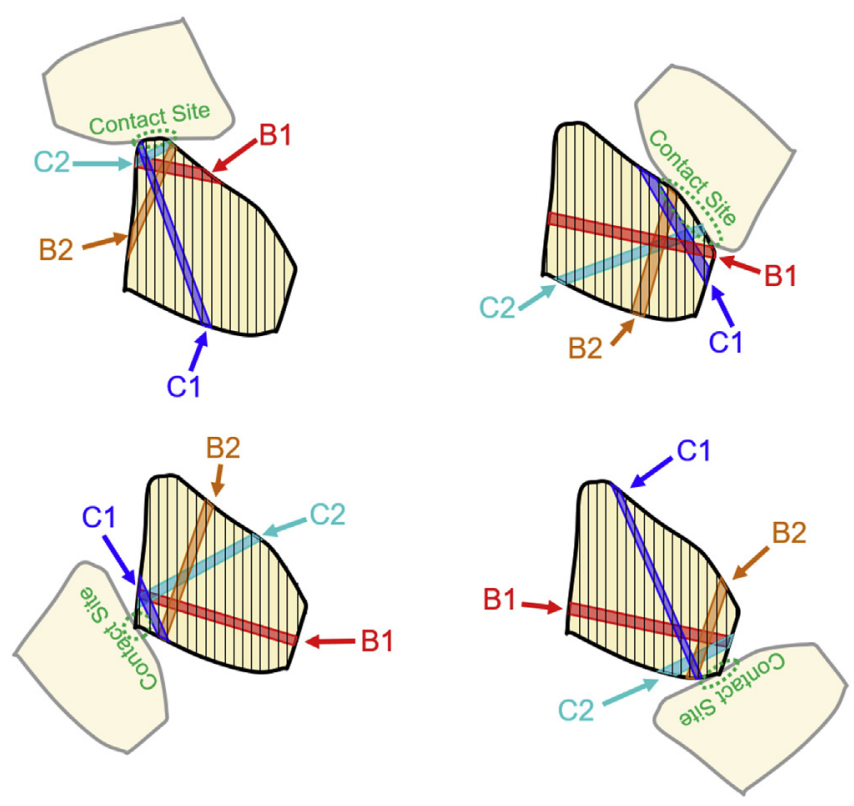

Fig. 12. Cross-sectional view of a generic single crystal austenitic shape memory ceramic particle with hatch lines indicating (001) direction. For each contact site, stress concentration may lead to formation of a martensite variant corresponding to one of the four ORs: B1, C1, B2, or C2.

packings martensite growth is limited inside individual singlecrystal particles. The size of each martensite plate is thus a fraction of the particle size. For a particle of a few microns in diameter and an interface propagating at the speed of sound in zirconia, the time required to form each martensite plate is on the order of $10^{-9} \mathrm{~s}$.

Based on these discussions, we propose the following rules for martensite nucleation and growth during SIMT in granular packings:

- Martensite nucleation will most likely occur close to the contact area and the surrounding free surface. For each contact site, the nucleated plate corresponds to one of the four ORs that most relieves the local strain energy. The number of martensite plates inside each single crystal particle depends on its contact coordination number $Z$.

- Martensite growth is fundamentally limited by the isolated nature of each particle in the packing. It will likely stop at the free surface of the particle. Once nucleated at a given load $P$, the martensite grows very rapidly and reaches the final thickness almost instantaneously.

\subsection{Evolution of the total number of martensite plates with load}

We next discuss how the external load influences the nucleation events and the number of martensite plates in granular packings. According to experimental observation and modeling of granular mechanics [20,64,65], regardless of the macroscopic loading condition (e.g. confined compression or simple shear), there is always a distribution of force $F$ carried by the individual particles, which is dependent on the external load $P$. In many cases, the force distribution follows a power law below the average value and resembles exponential decay above the average value $[20,66,67]$ :

$p(F(P))= \begin{cases}k_{1} F^{-a_{1}}+B_{1} \\ k_{2} \exp \left[-a_{2} F\right]+B_{2}, & F \geq\langle F\rangle\end{cases}$
Here, $k_{1}, k_{2}, B_{1}$, and $B_{2}$ are fitting parameters that adjust the scale of the probability distribution, while $a_{1}$ and $a_{2}$ are shape parameters. These parameters are defined such that at the average force value $\langle F>, p(F(P))$ is smooth and $d p / d F(P)$ is continuous. As the applied load $P$ increases, the average force $\langle F\rangle$ increases, $p(F(P))$ increases for high $F$ values, and particles bearing low or zero force become less likely. The probability distribution $p(F(P))$ thus becomes flatter - more heterogeneous - as the load is increased. The force distribution and its change with external load $P$ are schematically shown in Fig. 13.

The level of stress concentration close to the contact sites strongly depends on the magnitude of the force carried by individual particles. For example, with normal contact between solids of resolution, the maximum equivalent stress scales with the cube root of contact force, $\sigma_{e} \propto F^{\frac{1}{3}}$ [23]. It is thus natural to extend the critical stress concept for transformation in a monolithic solid to the concept of critical force carried by individual particles in a granular packing. For particles of a given size and composition, at a given temperature, we may assume that there is a critical force for martensite nucleation $F_{C}^{\text {Forward }}$ (the superscript denotes forward transformation), and the martensite nucleation is only possible in particles carrying normal force $F>F_{C}^{\text {Forward }}$. These particles are part of the strong force network and form 'transformation chains.' While this assumption of a critical force for transformation is exactly right only for the ideal case of Hertzian contact, it is still reasonable as a first-order approximation in modeling the nonideal case of our experiments-in which both tangent and normal forces are carried by the particles and SIMT is governed by both shear and dilatational stress components $[7,8]$.

It is important to note that $F_{C}^{\text {Forward }}$ is dependent on temperature, with higher temperatures causing an increase in the magnitude of force necessary to generate critical stress $\sigma_{C}$. This is because of the Clausius-Clapeyron relation $\frac{d \sigma_{C}}{d T}=-\frac{1}{\varepsilon^{\text {Tran }}} \frac{\Delta H}{T}$, where $\varepsilon^{\text {Tran }}$ is the transformation strain, and $\Delta H$ is the enthalpy of the transformation per unit volume. For the case of zirconia, SIMT releases heat $(\Delta H<0)$ and leads to volume increase $\left(\varepsilon^{\operatorname{Tran}}>0\right)$. Therefore, $\frac{d \sigma_{c}}{d T}>0$. In other words, if temperature increases in $\mathrm{ZrO}_{2}-\mathrm{CeO}_{2}$, the critical stress $\sigma_{C}$ and the critical force for transformation $F_{C}^{\text {Forward }}$ will both

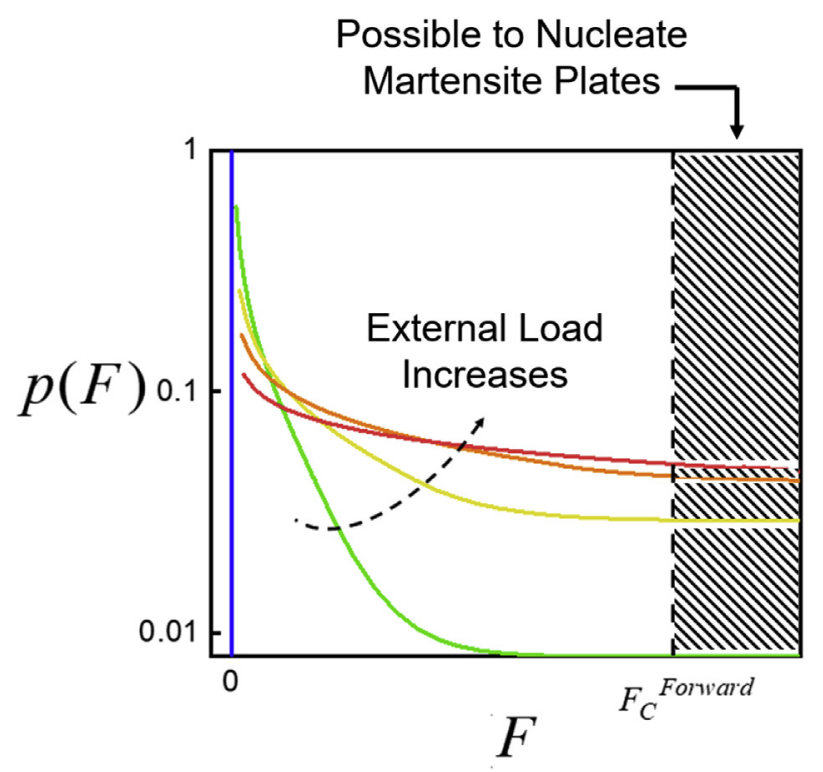

Fig. 13. An example of the probability density function for force distribution in a granular packing and its evolution with increasing loads. Higher loads increase the probability of a particle carrying force above $F_{C}^{\text {Forward }}$, thereby increasing the number of martensite plates in the packing. 
increase. For the temperature range of interest in this work (e.g. up to a few hundreds of Kelvin), there is no significant change of the stiffness or strength of $\mathrm{ZrO}_{2}-\mathrm{CeO}_{2}$ particles. At the same macroscopic load, therefore, the shape of $p(F(P))$ should remain almost the same. However, because $F_{C}^{\text {Forward }}$ increases at higher temperature, the fraction of particles in the transformation chains, which is determined by the area beneath the $p(F(P))$ curve and to the right of $F_{C}^{\text {Forward }}$, will decrease.

With the critical force assumption, the fraction of particles that can form martensite plates is $\int_{F_{\text {Forward }}}^{\infty} p(F) d F$ (see Fig. 13). Inside each of these particles, the number of martensite plates depends on the contact coordination number $Z$. It is possible that these particles have contacts in addition to the two strong contacts that make them in the transformation chain. If the additional contacts are weak, no martensite plates will form close to these contacts. Therefore, the number of martensite plates in each particle in the transformation chains may be expressed as $\eta Z$, where $\eta(0<\eta<1)$ is a parameter that accounts for the average probability of nucleating a martensite at a given contact. If the total particle number is $N_{\text {Total }}$, then the total number of formed martensite plates $N_{\text {Plate }}$ is

$N_{\text {Plate }}=\eta Z N_{\text {Total }} \int_{F_{C}^{\text {Forward }}}^{\infty} p(F) d F$

Then what happens as the external load $P$ slightly increases during loading? There will be a shift of the force distribution curve toward the large force direction, so more particles will carry the force $F \geq F_{C}^{\text {Forward }}$. This will lead to more nucleation events to form new martensite plates. When the external load increases from $P$ to $P+\Delta P$, the increase of martensite plate number will be $\Delta N_{\text {Plate }}=$ $\triangle P \eta Z N_{\text {Total }} \int_{F_{C}^{\text {Forward }}}^{\infty}[d p(F) / d P] d F$. This physical picture is illustrated in
Fig. 14, which shows that SIMT in a granular packing is nucleation controlled and the increase of martensite phase is by nucleating new martensite plates in individual particles. To compare, in the case of a monolithic single crystal or oligocrystal under uniaxial loading, the stress distribution is much more uniform, which facilitates easier martensite growth than additional martensite nucleation during deformation. As a result, the martensite fraction mainly increases by interface migration without a significant increase of the number of martensite plates [68].

What happens as the external load $P$ slightly decreases during unloading? There will be a shift of the force distribution curve toward the small force direction. Reverse transformation during unloading requires a lower critical stress than forward transformation, so the critical force for reverse transformation $F_{C}^{\text {Reverse }}<$ $F_{C}^{\text {Forward }}$. Reverse transformation will occur in particles that originally carried the force of $F \geq F_{C}^{\text {Forward }}$, but now reduce their carried force to $F^{\prime}<F_{C}^{\text {Reverse }}$. From granular mechanics experiments, a slight decrease of the macroscopic load often results in a number of particles completely dropping their load and becoming part of the weak network [21,53]. At every discrete instance where $F^{\prime}<F_{C}^{\text {Reverse }}$, the free energy of martensite plates will immediately become higher than the untransformed austenite. This will lead to rapid migration of the austenite-martensite interface and a complete reverse transformation. As a result, the martensite plates will be eliminated at the free surface in these particles, and the total number of martensite plates in the packing will decrease. We note that the above discussion is applicable to the $\mathrm{ZrO}_{2}-15$ at\% $\mathrm{CeO}_{2}$ packing, but not the $\mathrm{ZrO}_{2}-12$ at $\% \mathrm{CeO}_{2}$ packing. The latter is associated with the metastable austenite phase; even with the force distribution shift during unloading, no reverse transformation will occur. (a)

$$
\begin{gathered}
\text { Load 1 } \\
\langle F>\sim \text { zero } \\
N_{\text {Plates }} \sim \text { zero }
\end{gathered}
$$

Load 2

$<F>$ low

$N_{\text {Plates }}$ few

$V_{\text {Plate }}$ low

Load 3

$\langle F\rangle$ moderate

$N_{\text {Plates }}$ more

$V_{\text {Plate }}$ moderate

Load 4

$<F>$ high

$N_{\text {Plates }}$ many

$V_{\text {Plate }}$ high
Force Chain Martensite Phase
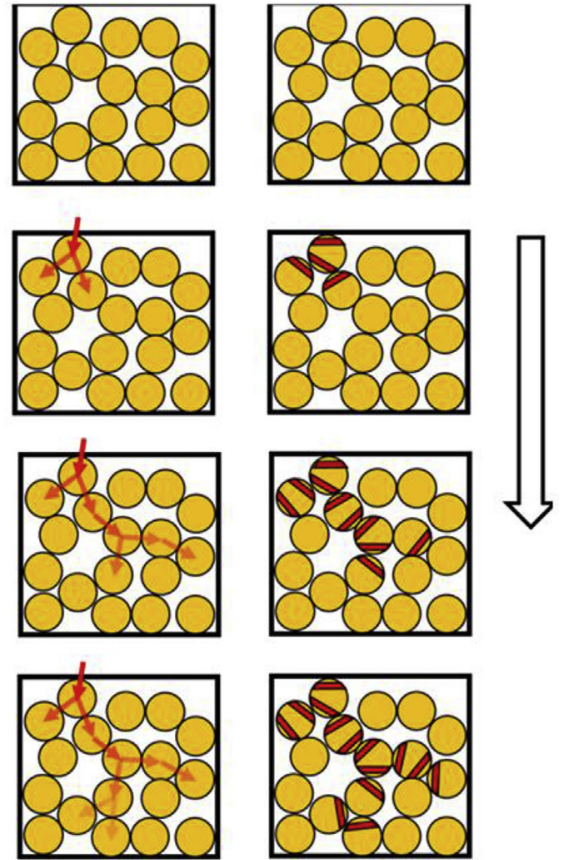

(b)

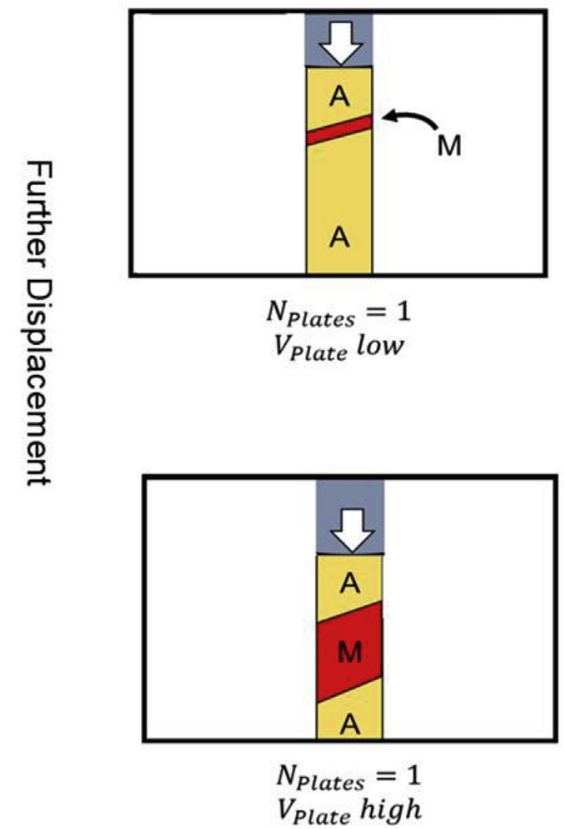

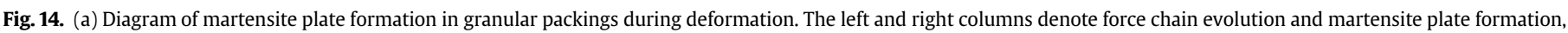

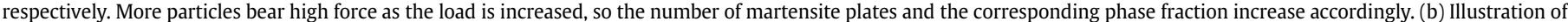

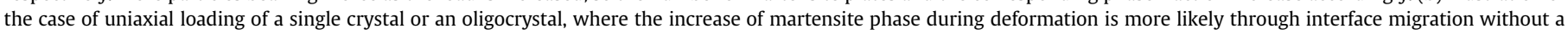
significant increase of the number of martensite plates. 


\subsection{Explanation of experimental observations in this work}

The discussions in Sections 6.1 and 6.2 can explain the trends observed from in situ neutron diffraction. First, the continuous transformation mode observed in both the $\mathrm{ZrO}_{2}-12$ at\% $\mathrm{CeO}_{2}$ (Fig. 3) and $\mathrm{ZrO}_{2}-15$ at $\% \mathrm{CeO}_{2}$ packings (Fig. 7) can be explained by the wide distribution of force among particles and the continuous shift of the force distribution curve with external load. According to Equation (2), a small increase of the macroscopic load $\Delta P$ will lead to nucleation of new martensite plates $\left(\Delta N_{\text {Plate }} \propto \Delta P Z N_{\text {Total }} \int_{F_{C}^{\text {Forward }}}^{\infty}[d p(F) / d P] d F\right)$, and therefore an increase of the transformed volume. As a result, although there is a critical force $F_{C}^{\text {Forward }}$ for transformation of individual particles, the whole granular packing shows a macroscopically continuous transformation mode in response to the external load, which is in striking contrast with the case of monoclinic solids.

Second, the peak broadness evolution can be explained by the formation and elimination of martensite plates, which lead to an increase and decrease of the interface area, respectively. As discussed in Section 5.2, the glissile interface between the austenitemartensite is conjected to consist of a dislocation array. This indeed has been observed by TEM for $\mathrm{ZrO}_{2}-3 \mathrm{~mol} \% \mathrm{Y}_{2} \mathrm{O}_{3}$, where an interface between the monoclinic and tetragonal structures can have as many as one dislocation per 33 (001) tetragonal atom planes [59]. The interfaces are thus associated with micro-strain distributions, and the area of all interfaces has an influence on the associated peak broadness. During loading in both $\mathrm{ZrO}_{2}-12$ at\% $\mathrm{CeO}_{2}$ and $\mathrm{ZrO}_{2}-15$ at\% $\mathrm{CeO}_{2}$ packings, new martensite plates form and each new plate has at least one strained interface with the austenite. As a result, the peak broadness increases monotonically with load. During unloading of the $\mathrm{ZrO}_{2}-15$ at $\% \mathrm{CeO}_{2}$ packing, the reverse transformation is associated with elimination of martensite plates and decrease of the interface area. The dislocations once populating the interface are absorbed by the free surface on which the plate is originally nucleated. As a result, the peak broadness continuously decreases and eventually returns to its original value. During unloading of the $\mathrm{ZrO}_{2}-12 \mathrm{at} \% \mathrm{CeO}_{2}$ packing, no reverse transformation occurs, no interface migration occurs, and the peak broadness stays almost constant, although the average lattice strain drops to zero. These prediction results are consistent with the data in Figs. 9 and 10.

\section{Conclusions}

We have explored SIMT in granular $\mathrm{ZrO}_{2}-12$ at $\% \mathrm{CeO}_{2}$ and $\mathrm{ZrO}_{2}-$ 15 at\% $\mathrm{CeO}_{2}$ packings through in situ neutron diffraction. We have also discussed the mechanism of martensite nucleation and growth in granular packings and have explained the experimental observations accordingly. The most salient conclusions from this work are:

- The $\mathrm{ZrO}_{2}-12$ at\% $\mathrm{CeO}_{2}$ packing shows SIMT during loading, no phase transformation during unloading, and thermally-induced reverse transformation during subsequent heating. The $\mathrm{ZrO}_{2}-$ 15 at\% $\mathrm{CeO}_{2}$ packing shows SIMT during loading and reverse transformation during unloading. This is the first direct evidence that reversible SIMT can occur in granular materials.

- Compression of a loose shape memory ceramic packing involves multiple stages in sequence, defined by particle rearrangement, contact deformation, and simultaneous contact deformation and martensitic transformation.

- SIMT in granular packings shows a continuous mode without a critical load and with a wide range of transformation loads. This is a result of the wide distribution of force carried by individual particles, wherein a slight increase of the external load can cause more particles to carry forces above the threshold for martensite nucleation.

- The martensitic transformation is nucleation-controlled and its growth is limited by the particle size. The strong correlation between the peak broadening and the transformed phase fraction can be explained by the increase or decrease of the austenite-martensite interface area during formation or elimination of martensite plates.

In this work, potential interfering effects from plasticity and intra-particle mechanical constraints are prevented by using packings that consist of single crystal $\mathrm{ZrO}_{2}-\mathrm{CeO}_{2}$ micro-particles. Therefore, we expect the observed transformation characteristics to mostly reflect the heterogenous driving force and low barrier for martensite nucleation, which are a result of the special structural arrangement in granular materials.

\section{Acknowledgement}

Neutron diffraction work was carried out at the Spallation Neutron Source (SNS), which is the U.S. Department of Energy, United States user facility at the Oak Ridge National Laboratory, United States, sponsored by the Scientific User Facilities Division, Office of Basic Energy Sciences. The authors thank Mr. M. J. Frost at SNS for the technique support. The authors thank Mr. D. Garcia and Mr. R. J. Griffiths from Virginia Tech for helping with the in situ neutron diffraction experiment.

\section{Appendix A. Supplementary data}

Supplementary data to this article can be found online at https://doi.org/10.1016/j.actamat.2019.02.028.

\section{References}

[1] G.B. Olson, M. Cohen, A perspective on martensitic nucleation, Annu. Rev. Mater. Sci. 11 (1) (1981) 1-32.

[2] K. Otsuka, C.M. Wayman, Shape Memory Materials, Cambridge University Press, New York, 1998.

[3] Z.G. Wei, R. Sandstrom, S. Miyazaki, Shape memory materials and hybrid composites for smart systems: Part II Shape-memory hybrid composites, J. Mater. Sci. 33 (15) (1998) 3763-3783.

[4] Z.G. Wei, R. Sandstroröm, S. Miyazaki, Shape-memory materials and hybrid composites for smart systems: Part I Shape-memory materials, J. Mater. Sci. 33 (15) (1998) 3743-3762.

[5] D.C. Dunand, P. Müllner, Size effects on magnetic actuation in Ni-Mn-Ga shape-memory alloys, Adv. Mater. 23 (2) (2011) 216-232.

[6] M. Chmielus, X.X. Zhang, C. Witherspoon, D.C. Dunand, P. Müllner, Giant magnetic-field-induced strains in polycrystalline $\mathrm{Ni}-\mathrm{Mn}-\mathrm{Ga}$ foams, Nat. Mater. 8 (2009) 863.

[7] A.H. Heuer, M. Rühle, Overview no. 45, Acta Metall. 33 (12) (1985) $2101-2112$

[8] W.M. Kriven, Martensitic toughening of ceramics, Mater. Sci. Eng. 127 (2) (1990) 249-255.

[9] A.G. EVANS, A.H. HEUER, REVIEW-transformation toughening in ceramics: martensitic transformations in crack-tip stress fields, J. Am. Ceram. Soc. 63 (56) (1980) 241-248.

[10] P.E. REYES-MOREL, J.-S. CHERNG, I.-W. CHEN, Transformation plasticity of CeO2-stabilized tetragonal zirconia polycrystals: II, pseudoelasticity and shape memory effect, J. Am. Ceram. Soc. 71 (8) (1988) 648-657.

[11] C. Chluba, W. Ge, R. Lima de Miranda, J. Strobel, L. Kienle, E. Quandt M. Wuttig, Ultralow-fatigue shape memory alloy films, Science 348 (6238) (2015) 1004.

[12] Z. Du, X.M. Zeng, Q. Liu, C.A. Schuh, C.L. Gan, Superelasticity in micro-scale shape memory ceramic particles, Acta Mater. 123 (2017) 255-263.

[13] S.M. Ueland, C.A. Schuh, Superelasticity and fatigue in oligocrystalline shape memory alloy microwires, Acta Mater. 60 (1) (2012) 282-292.

[14] A. Lai, Z. Du, C.L. Gan, C.A. Schuh, Shape memory and superelastic ceramics at small scales, Science 341 (6153) (2013) 1505.

[15] P.M. Kelly, L.R. Francis Rose, The martensitic transformation in ceramics - its role in transformation toughening, Prog. Mater. Sci. 47 (5) (2002) 463-557.

[16] I.W. Chen, Implications of transformation plasticity in ZrO2-containing ceramics: II, elastic-plastic indentation, J. Am. Ceram. Soc. 69 (3) (1986) 189-194. 
[17] P.E. Reyes-Morel, J.-S. Cherng, I.W. Chen, Transformation plasticity of CeO2stabilized tetragonal zirconia polycrystals: II, pseudoelasticity and shape memory effect, J. Am. Ceram. Soc. 71 (8) (1988) 648-657.

[18] P. Claudin, J.P. Bouchaud, M.E. Cates, J.P. Wittmer, Models of stress fluctuations in granular media, Phys. Rev. 57 (4) (1998) 4441-4457.

[19] R.C. Hidalgo, C.U. Grosse, F. Kun, H.W. Reinhardt, H.J. Herrmann, Evolution of percolating force chains in compressed granular media, Phys. Rev. Lett. 89 (20) (2002) 205501.

[20] F. Radjai, M. Jean, J.-J. Moreau, S. Roux, Force distributions in dense twodimensional granular systems, Phys. Rev. Lett. 77 (2) (1996) 274-277.

[21] T.S. Majmudar, R.P. Behringer, Contact force measurements and stressinduced anisotropy in granular materials, Nature 435 (2005) 1079.

[22] A. Tordesillas, S.T. Tobin, M. Cil, K. Alshibli, R.P. Behringer, Network flow model of force transmission in unbonded and bonded granular media, Phys. Rev. 91 (6) (2015) 062204.

[23] K.L. Johnson, Contact Mechanics, Cambridge University, 1987. Press.

[24] X. Zeng, Z. Du, C. Schuh, C. Gan, Enhanced shape memory and superelasticity in small-volume ceramics: a perspective on the controlling factors, MRS Communications 7 (4) (2017) 747-754, https://doi.org/10.1557/mrc.2017.99.

[25] Z. Du, P. Ye, X.M. Zeng, C.A. Schuh, N. Tamura, X. Zhou, C.L. Gan, Synthesis of monodisperse $\mathrm{CeO} 2-\mathrm{ZrO} 2$ particles exhibiting cyclic superelasticity over hundreds of cycles, J. Am. Ceram. Soc. 100 (9) (2017) 4199-4208.

[26] X.M. Zeng, Z. Du, N. Tamura, Q. Liu, C.A. Schuh, C.L. Gan, In-situ studies on martensitic transformation and high-temperature shape memory in small volume zirconia, Acta Mater. 134 (2017) 257-266.

[27] I.-W. Chen, L.A. Xue, Development of superplastic structural ceramics, J. Am. Ceram. Soc. 73 (9) (1990) 2585-2609.

[28] H.Z. Yu, M. Hassani-Gangaraj, Z. Du, C.L. Gan, C.A. Schuh, Granular shape memory ceramic packings, Acta Mater. 132 (2017) 455-466.

[29] P.R. Brewin, Modelling of powder die compaction, Springer, London, 2008.

[30] A. Stebner, X. Gao, D.W. Brown, L.C. Brinson, Neutron diffraction studies and multivariant simulations of shape memory alloys: empirical texture development-mechanical response relations of martensitic nickel-titanium, Acta Mater. 59 (7) (2011) 2841-2849.

[31] A.P. Stebner, H.M. Paranjape, B. Clausen, L.C. Brinson, A.R. Pelton, In situ neutron diffraction studies of large monotonic deformations of superelastic nitinol, Shape Memory and Superelasticity 1 (2) (2015) 252-267.

[32] G. Esteves, C.M. Fancher, J.L. Jones, In situ characterization of polycrystalline ferroelectrics using x-ray and neutron diffraction - ERRATUM, J. Mater. Res. 30 (3) (2015), 451-451.

[33] Y. Ma, E.H. Kisi, S.J. Kennedy, Neutron diffraction study of ferroelasticity in a 3 mol\% Y2O3-ZrO2, J. Am. Ceram. Soc. 84 (2) (2001) 399-405.

[34] Y. Chen, D.J. Yu, K. An, Stress-induced charge-ordering process in LiMn2O4, Materials Research Letters 5 (2) (2017) 89-94.

[35] H.M. Paranjape, P.P. Paul, B. Amin-Ahmadi, H. Sharma, D. Dale, J.Y.P. Ko, Y.I. Chumlyakov, L.C. Brinson, A.P. Stebner, In situ, 3D characterization of the deformation mechanics of a superelastic NiTi shape memory alloy single crystal under multiscale constraint, Acta Mater. 144 (2018) 748-757.

[36] K. An, H.D. Skorpenske, A.D. Stoica, D. Ma, X.-L. Wang, E. Cakmak, First in situ lattice strains measurements under load at VULCAN, Metall. Mater. Trans. 42 (1) (2011) 95-99.

[37] C. Shang, I.C. Sinka, J. Pan, Constitutive model calibration for powder compaction using instrumented die testing, Exp. Mech. 52 (7) (2012) 903-916.

[38] K. An, VDRIVE - Data Reduction and Interactive Visualization Software fo Event Mode Neutron Diffraction, ORNL Report, ORNL-TM-2012-621, Oak Ridge National Laboratory, 2012.

[39] H.M. Rietveld, A profile refinement method for nuclear and magnetic structures, J. Appl. Crystallogr. 2 (2) (1969) 65-71.

[40] A.C.L.a.R.B.v. Dreele, General structure analysis system (GSAS), Los Alamos National Laboratory Report LAUR (2004) 86-748.

[41] B.H. Toby, EXPGUI, a graphical user interface for GSAS, J. Appl. Crystallogr. 34 (2) (2001) 210-213.

[42] P.E. REYES-MOREL, I.-W. CHEN, Transformation plasticity of CeO2-stabilized tetragonal zirconia polycrystals: I, stress assistance and autocatalysis, J. Am. Ceram. Soc. 71 (5) (1988) 343-353.
[43] M.X. Lim, R.P. Behringer, Topology of Force Networks in Granular Media under Impact, 2017.

[44] L. Papadopoulos, J.G. Puckett, K.E. Daniels, D.S. Bassett, Evolution of network architecture in a granular material under compression, Phys. Rev. 94 (3) (2016) 032908.

[45] F. Radjai, M. Jean, J.J. Moreau, S. Roux, Force distributions in dense twodimensional granular systems, Phys. Rev. Lett. 77 (2) (1996) 274-277.

[46] Y. Zhang, U. Duke, R. Behringer, U. Duke, Pulling Out an Intruder, 2017. Retrieved, https://webhome.phy.duke.edu/ bob/. (Accessed 9 January 2019).

[47] Y. Zhang, R. Behringer, Pulling an intruder from a granular material : a novel depinning experiment, Powders \& Grains 03040 (2017) 4-7.

[48] F. Göncü, O. Durán, S. Luding, Constitutive relations for the isotropic deformation of frictionless packings of polydisperse spheres, Compt. Rendus Mec. $338(10-11)(2010)$ 570-586.

[49] O.I. Imole, M. Wojtkowski, V. Magnanimo, S. Luding, Micro-macro correlations and anisotropy in granular assemblies under uniaxial loading and unloading, Phys. Rev. E - Stat. Nonlinear Soft Matter Phys. 89 (4) (2014) 1-23.

[50] M. Yashima, T. Mitsuhashi, H. Takashina, M. Kakihana, T. Ikegami, M. Yoshimura, Tetragonal-monoclinic phase transition enthalpy and temperature of $\mathrm{ZrO} 2-\mathrm{CeO} 2$ solid solutions, J. Am. Ceram. Soc. 78 (8) (1995) 2225-2228.

[51] H.F. Fischmeister, E. Arzt, Densification of powders by particle deformation, Powder Metall. 26 (2) (1983) 82-88.

[52] A.S. Helle, K.E. Easterling, M.F. Ashby, Hot-isostatic pressing diagrams: new developments, Acta Metall. 33 (12) (1985) 2163-2174.

[53] E.T. Bowman, K. Soga, Creep, ageing and microstructural change in dense granular materials, Soils Found. 43 (4) (2003) 107-117.

[54] P. Scardi, R.D. Maggio, L. Lutterotti, P. Maistrelli, Thermal expansion anisotropy of ceria-stabilized tetragonal zirconia, J. Am. Ceram. Soc. 75 (10) (1992) 2828-2832.

[55] J.L. Jones, M. Hoffman, S.C. Vogel, Ferroelastic domain switching in lead zirconate titanate measured by in situ neutron diffraction, Mech. Mater. 39 (4) (2007) 283-290.

[56] J.S. Forrester, E.H. Kisi, A.J. Studer, Direct observation of ferroelastic domain switching in polycrystalline BaTiO3 using in situ neutron diffraction, J. Eur. Ceram. Soc. 25 (4) (2005) 447-454.

[57] D.A. Porter, K.E. Easterling, M.Y. Sherif, Phase Transformations in Metals and Alloys, third ed., CRC Press, Boca Raton, FL, 2009.

[58] J.W. Christian, G.B. Olson, M. Cohen, Classification of displacive transformations: what is a martensitic transformation? J. Phys. IV 5 (C8) (1995) $3-10$.

[59] W.Z. Zhu, T.C. Lei, Y. Zhou, Z.S. Ding, HREM investigation of interface between tetragonal and monoclinic phases in a $\mathrm{ZrO} 2(3 \mathrm{~mol} \% \mathrm{Y} 2 \mathrm{O} 3)$ ceramic, J. Mater. Sci. Lett. 15 (1) (1996) 69-71.

[60] P.C. Clapp, A localized soft mode theory for martensitic transformations, Phys. Status Solidi 57 (2) (1973) 561-569.

[61] G. GuÉnin, P.F. Gobin, A localized soft mode model for the nucleation of thermoelastic martensitic transformation: application to the $\beta \rightarrow$ 9r transformation, Metallurgical Transactions A 13 (7) (1982) 1127-1134.

[62] S. Kajiwara, Metall. Mater. Trans. 17 (1986) 1693. https://doi.org/10.1007/ BF02817268.

[63] K. Momma, F. Izumi, VESTA 3 for three-dimensional visualization of crystal, volumetric and morphology data, J. Appl. Crystallogr. 44 (6) (2011) $1272-1276$.

[64] F. Radjai, F. Dubois, Discrete-element Modeling of Granular Materials, WileyIste, 2011.

[65] E. Azéma, F. Radjaï, Stress-strain behavior and geometrical properties of packings of elongated particles, Phys. Rev. 81 (5) (2010) 051304

[66] F. Radjai, D.E. Wolf, M. Jean, J.-J. Moreau, Bimodal character of stress transmission in granular packings, Phys. Rev. Lett. 80 (1) (1998) 61-64.

[67] N.P. Kruyt, On weak and strong contact force networks in granular materials, Int J. Solids Struct 92-93 (2016) 135-140.

[68] S.M. Ueland, C.A. Schuh, Transition from many domain to single domain martensite morphology in small-scale shape memory alloys, Acta Mater. 61 (15) (2013) 5618-5625. 